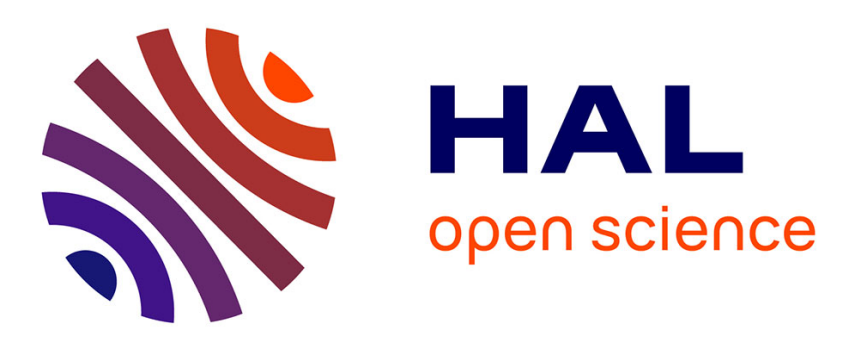

\title{
Crystallization kinetics of polymer fibrous nanocomposites
}

\author{
Stanislaw Galeski, Ewa Piórkowska, Artur Rozanski, Gilles Regnier, Andrzej \\ Galeski, Kinga Jurczuk
}

\section{To cite this version:}

Stanislaw Galeski, Ewa Piórkowska, Artur Rozanski, Gilles Regnier, Andrzej Galeski, et al.. Crystallization kinetics of polymer fibrous nanocomposites. European Polymer Journal, 2016, 83, pp.181-201. 10.1016/j.eurpolymj.2016.08.002 . hal-02291285

\section{HAL Id: hal-02291285 \\ https://hal.science/hal-02291285}

Submitted on 18 Sep 2019

HAL is a multi-disciplinary open access archive for the deposit and dissemination of scientific research documents, whether they are published or not. The documents may come from teaching and research institutions in France or abroad, or from public or private research centers.
L'archive ouverte pluridisciplinaire HAL, est destinée au dépôt et à la diffusion de documents scientifiques de niveau recherche, publiés ou non, émanant des établissements d'enseignement et de recherche français ou étrangers, des laboratoires publics ou privés. 


\title{
Macromolecular Nanotechnology
}

\section{Crystallization kinetics of polymer fibrous nanocomposites}

\author{
Stanislaw Galeski ${ }^{1}$, Ewa Piorkowska*, Artur Rozanski, Gilles Regnier ${ }^{2}$, Andrzej Galeski, \\ Kinga Jurczuk
}

Centre of Molecular and Macromolecular Studies, Polish Academy of Sciences, 90-363 Lodz, Poland

Keywords:

Kinetics of crystallization

Nanocomposites

Fibrillar reinforcement

Extended volume

Probability approach

\begin{abstract}
A B S T R A C T
Through applying both a probabilistic approach and a combination of probabilistic and the Avrami 'extended volume' approaches we have derived a theory of overall crystallization kinetics of polymers reinforced with nanofibers. The theory describes the crystallization kinetics in the presence of straight or curved nanofibers, with different nucleation ability and orientation, and allows to account for their variable length. The analytic results are supported by computer simulations of spherulitic structures. The derived mathematical formulas are in exponential forms suggesting the use of the Avrami logarithmic coordinates for detailed analysis of experimental data. Experimental data on crystallization of several nanocomposites, including polypropylene reinforced with poly(tetrafluoroethylene) nanofibers and polyamide 12 with carbon nanotubes, are in a good agreement with the theoretical predictions.
\end{abstract}

\section{Introduction}

There are many well known advantages of reinforcing polymers with fibers. The use of nanofibers as fillers permits to achieve those goals at very low loading levels, usually a few percent. Polymer nanocomposites comprise also materials where the reinforcement can be polymeric. In self-reinforced polymer materials, also referred to as single-phase or homocomposites, the same polymer is forming both the reinforcing and matrix phases.

Fibers can strongly affect not only the properties but also crystallization of composites because of the presence of internal interfaces. Fibers frequently exhibit nucleation activity. Acting as a nucleating agent they shorten the crystallization time and increase the temperature at which the polymer crystallizes during processing, thus reducing cycle time and increasing productivity. Fibrous fillers induce crystallization of the polymer matrix thorough nucleation on their surfaces. It appears that they can either nucleate very strongly causing the growth of transcrystalline layers around them [e.g. 1] or the nucleation activity can be limited though still accelerating crystallization [e.g. 2]. In several studies it was shown that agglomerates of fibrous nanofillers can efficiently nucleate crystallization while along a single nanofiber the crystallization is activated on several points only [3]. Polymer matrices that were studied include polypropylene [e.g. 4], polylactide [5], polycaprolactones [6,7], poly(ethylene terephthalate) [8], poly(butylene terephthalate) [9], syndiotactic polystyrene [10], and other. Among fibrous fillers were carbon nanotubes, both single and multi-wall [3,11-13], polymeric nanofibers [14,15], rodlike silicon nitride [4], and carbon nanofibers [16,17].

\footnotetext{
* Corresponding author.

E-mail address: epiorkow@cbmm.lodz.pl (E. Piorkowska).

1 Present address: Solid State Physics Laboratory, Department of Physics, ETH Zurich, CH-8093 Zurich, Switzerland.

2 PIMM, Arts et Métiers ParisTech, CNRS, CNAM, Paris, France.
} 
In most of the above cited reports the Avrami approach for the analysis of the kinetics of crystallization was applied without full justification. The original Avrami concept [18-20] was designed for random nucleation sites and radial growth of crystalline aggregates, whereas the nucleation sites on fiber surfaces are spatially correlated. In addition, in several above mentioned papers the isothermal Avrami treatment was erroneously applied to nonisothermal crystallization or to crystallization degree rather than to conversion degree. This indicates clearly that a coherent theory of crystallization kinetics of fibrous nanocomposites is lacking and is seriously needed. In this work we describe an effort to develop such theory basing on known approaches to crystallization kinetics of simpler systems.

\section{Basis of the theory}

The description of the overall crystallization kinetics in polymers was recently reviewed and assessed in [21,22]. There are two main approaches: one based on the Avrami [18-20] concept of the so called 'extended volume' and second applying probability theory first used by Evans [23]. The 'extended volume' at time $t$ is a sum of volumes of all domains neglecting the truncation upon impingement. The extended volume includes also the 'phantom' domains expanding from nucleation 'attempts' in already crystallized regions. At time $t$ the 'extended volume' fraction increment, $d E(t)$, occurring in the unconverted fraction, $1-\alpha(t)$, expressed as $d E(t)[1-\alpha(t)]$, is equal to the increment of real converted volume fraction, $d \alpha(t)$. After simple transformation and integration the conversion degree, $\alpha$, is expressed by the well-known Avrami equation:

$$
\alpha(t)=1-\exp [-E(t)]
$$

In a uniform temperature field $E$ can be easily calculated as $D V(0, t)$ and $\int_{0}^{t} F(\tau) V(\tau, t) d \tau$, for instantaneous nucleation with the density $D$ and spontaneous nucleation with the time dependent rate $F$ (a number of nuclei per a volume unit and time unit), respectively, and where $V(\tau, t)$ denotes the volume at time $t$ of the domain nucleated at $\tau$. Needless to say that in the case of isothermal conditions when the domain growth rate is constant, and for instantaneous nucleation or spontaneous nucleation with a constant rate, $E$ in the form of $K t^{n}$ is obtained.

The derivations based on probability theory utilize the formula describing the probability that a number of nucleation events will occur in a finite volume. In the derivations based on the probabilistic approach the key point is to calculate the probability that an arbitrarily chosen point, denoted here as $A$, at a given time $t$, will remain outside growing domains, $P_{0}(t)$, that enables to calculate $\alpha$ as:

$$
\alpha(t)=1-P_{0}(t)
$$

where $P_{0}$ is equal to $\exp (-E)$, where $E$ is an expected value of the Poisson probability distribution. $P_{0}(t)$ is in fact the probability that no nucleation event will occur in an appropriate time intervals in corresponding finite volumes around $A$. Evans [23] calculated $E$ for the constant growth rate of domains, and for instantaneous and spontaneous nucleation with a constant rate, obtaining $E$ in the form of $K t^{n}$. Further development of the theory was focused on time dependent nucleation rate [24], nonisothermal crystallization [25], and utilizing the concept of nucleation as a set of events occurring randomly not only in space but also in time [26]. The latter allowed to calculate $\alpha$, in a relatively simple way, for complex crystallization conditions, such as crystallization in a temperature gradient [27]. Recently, kinetic model of development of lamellar thickness distribution and average lamellar thickness during melt crystallization was proposed based on the Avrami-Evans approach [28].

The limits of applicability of the both approaches were recently discussed in [21,22]. It must be noted that for crystallization in an infinite volume in a uniform temperature field the same equations are obtained regardless whether the "extended volume' approach or the probabilistic approach was used. However, if the conditions vary locally, and thus the nucleation rate or the growth rate varies, different expressions can be obtained [21] and calculations of the extended volume as a sum of volumes of all domains leads to a wrong result. These discrepancies vanish if the 'extended volume' is defined as the average number of extended domains that have grown through the point $A(x, y, z)$. $E(t, A)$ calculated for $A(x, y, z)$ becomes a function of spatial coordinates [20] as the expected value obtained through the probabilistic approach.

In the description of overall crystallization kinetics in a finite volume one has to consider that the area beyond boundaries of a polymer portion is not accessible for crystallization and that additional nucleation can occur on the boundaries $[21,22]$. Similar problems are encountered in the modelling of overall crystallization kinetics in fiber reinforced composites; the volume inhabited by fibers is not accessible for crystallization and spherulites can nucleate not only in the polymer bulk but also on the fibers surfaces; the intense nucleation on the fiber surface results in transcrystallinity [29]. The main difference is that fibers are dispersed within a polymer matrix in a more or less random way.

Only few attempts to model crystallization in fiber reinforced composites were undertaken so far. Computer modelling of isothermal crystallization in such systems concerned non-overlapping long fibers of equal diameters aligned parallel in one direction [30-33] dispersed randomly or arranged in a hexagonal array.

To predict the conversion of melt into spherulites during isothermal crystallization of fiber reinforced composites, the concept of 'extended volume' was used [34], but also the probabilistic approach was applied to bulk crystallization in composites reinforced with long fibers [33]. It was also assumed that the length of fibers exceeded significantly the spherulite size, therefore the effect of fiber ends could be neglected. 
The presence of fibers that did not nucleate crystallization decreased the overall crystallization rate in comparison to that of pure polymer, whereas the nucleation on fibers accelerated the crystallization [33]. Moreover, the slopes of the Avrami plots differed from those predicted by the Avrami-Evans theory for neat polymers, or even deviated from straight lines. The influence of fibers on overall crystallization rate increased with increasing fiber content and also with decreasing fiber diameter [33].

Similarly to fibers, reinforcing nanofibers are able or unable to nucleate matrix crystallization. Even weakly nucleating nanofibers can have strong effect on crystallization. The amplification of nucleating ability is connected with dramatic increase of the surface to volume ratio. It is well known that nanoparticles can also hinder the crystal growth rate [29]. However, if the nanofibers are themselves nucleation sites, their influence on the overall crystallization kinetics is dominated by their ability to nucleate.

This work focuses on modelling of overall crystallization kinetics in nanofiber reinforced composites based on the probabilistic and extended volume approaches. Nano-sized transversal sizes and small volume fraction of nanofibers allow to neglect their volume but the length of nanofibers is often too small to disregard the effect of nanofiber ends, as in the case of long fibers. In the following we first consider straight nanofibers of equal length with different nucleating ability. However, nanofibers in nanocomposites may have different length, curvature and orientation. Therefore the effect of length distribution and orientation is also elucidated. Moreover, curved nanofibers are also considered. The results of analytical models are verified by computer simulation and compared with experimental data.

\section{Straight nanofibers}

In order to simplify the problem, we first consider a nanocomposite with nanofibers of equal length, $2 L$, and all oriented in the same direction, and use the probabilistic approach to derive the expression for $\alpha$. The additional assumptions are:

(1) Crystallization occurs in 3D and in an infinite volume, which means that sizes of polycrystalline aggregates are at least several times smaller than sizes of the polymer portion.

(2) Crystallization of a polymer is spherulitic and isotropic, that is the nucleation rate, $F$ and the spherulite growth rate $G$, are independent of spatial coordinates. At time $t$ the 'extended' radius of a spherulite nucleated at $\tau$ is expressed by the equation:

$\rho(\tau, t)=\int_{\tau}^{t} G(s) d s$

(3) Thickness of nanofibers is negligibly small compared with spherulites sizes. $D_{f}$ denotes the number of nanofibers centers in a volume unit of the nanocomposite, and will be referred to as nanofiber content.

(4) Nucleation on nanofibers is instantaneous: all nuclei appear at the same time at the very beginning of crystallization. $D_{s}$ denotes the nucleation density on nanofibers - the number of nuclei per a nanofiber length unit.

\subsection{Probabilistic approach}

The key problem in the probabilistic approach to the overall crystallization kinetics is to calculate the probability, $P_{0}(t)$, that an arbitrarily chosen point will remain in the uncrystallized fraction of a material at time $t$, elapsed from the beginning of crystallization, that is from the moment of nucleation of the first spherulite. In the case of instantaneous nucleation, the point $A$ will remain outside of growing spherulites at time $t$, if none of them is nucleated within a sphere with the point $A$ at the center, and having the radius $R(t)=\rho(0, t) . P_{0}(t)$ is a product of the probability that no spherulite will be nucleated in a polymer bulk, denoted here as $P_{b}(t)$, and the probability that no spherulite will be nucleated on nanofibers, denoted as $P_{f}(t)$ :

$$
P_{0}(t)=P_{b}(t) P_{f}(t)
$$

According to the Avrami-Evans theory $P_{b}(t)$ equals:

$$
P_{b}(t)=\exp \left[-E_{b}(t)\right]
$$

with $E_{b}$ in the form:

$$
\begin{aligned}
& E_{b}(t)=(4 \pi / 3) D R(t)^{3} \\
& E_{b}(t)=(4 \pi / 3) \int_{0}^{t} F(\tau) \rho(\tau, t)^{3} d \tau
\end{aligned}
$$

for the instantaneous and spontaneous nucleation, respectively. In the case of the instantaneous nucleation on nanofibers, $P_{f}(t)$ is the probability that no nucleation event will occur on the nanofibers within a sphere around $A$, having the radius $R(t) . P_{f}(t)$ is a sum of the probability $P_{f a}$ that no nanofiber will cross the sphere around $A$ and the probability $P_{f b}$, that although any number of nanofibers cross the sphere, nothing will be nucleated on nanofiber sections embedded in the sphere. If all nanofibers are aligned in one direction, to fulfill the first condition nanofiber centers should be outside the zone of volume $(4 \pi / 3) R^{3}+2 \pi L R^{2}$ (Fig. 1 ), composed of a cylinder and two hemispheres. 

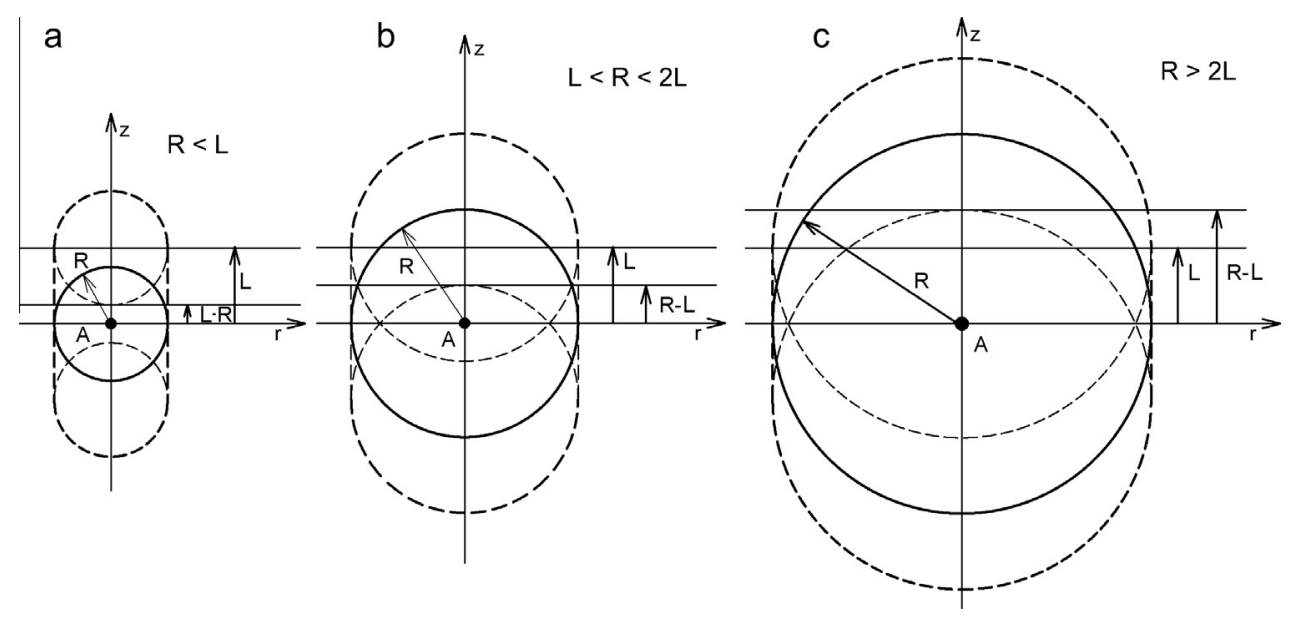

Fig. 1. Sphere of radius $R$ (solid line), and the zone composed of a cylinder and two hemispheres (dashed line) around the point $A$, for different ratios between $R$ and $L$.

According to the Poisson distribution, $P_{f a}$ is expressed as:

$$
P_{f a}(t)=\exp \left\{-D_{f}\left[(4 \pi / 3) R^{3}+2 \pi L R^{2}\right]\right\}
$$

Calculation of $P_{f b}$ is more complex and requires summation of the probabilities $P_{f b k}, k=1, \ldots, \infty$, that $k$ nanofibers will cross the sphere but no spherulite will be nucleated on their sections inside the sphere. Probability, that $k$ nanofibers aligned in $z-$ direction with centers located in the range of cylindrical coordinates between $\left(r_{i}, z_{i}\right)$ and $\left(r_{i}+d r_{i}, z_{i}+d z_{i}\right), i=1, \ldots, k$, will cross the sphere and nothing will be nucleated on their sections crossing the sphere, reads:

$$
p_{k}\left(t, r_{i}, z_{i}\right)=\exp \left\{-D_{f}\left[(4 \pi / 3) R^{3}+2 \pi L R^{2}\right]\right\} \prod_{i=1}^{k} D_{f} 2 \pi r_{i} p\left(r_{i}, z_{i}\right) d r_{i} d z_{i}
$$

where $p\left(r_{i}, z_{i}\right)=\exp \left[-D_{s} L_{c}\left(r_{i}, z_{i}\right)\right]$ denotes the probability that no nucleation event will occur on the nanofiber section $L_{c}\left(r_{i}, z_{i}\right)$ confined in the sphere, dependent on a position of the nanofiber relative to the point $A$. To calculate $P_{f b k}(t)$ it is necessary to integrate the products $r_{i} p\left(r_{i}, z_{i}\right)$ in Eq. (8) over $r_{i}$ and $z_{i}$ within the zone of volume $(4 \pi / 3) R^{3}+2 \pi L R^{2}$, and to divide the result by $k$ ! to eliminate multiple counting of the same events. Owing to the symmetry it is sufficient to limit to positive values of $z$ and to multiply the result by 2 . Finally:

$$
P_{f b k}(t)=\exp \left\{-D_{f}\left[(4 \pi / 3) R^{3}+2 \pi L R^{2}\right]\right\}\left[D_{f} C\left(R, D_{s}, L\right)\right]^{k}(1 / k !)
$$

where

$$
\begin{aligned}
& C\left(R, D_{s}, L\right)=4 \pi\left[\int_{0}^{L} \int_{0}^{R} r p(r, z) d r d z+\int_{L}^{L+R} \int_{0}^{U} r p(r, z) d r d z\right] \\
& U=\left[R^{2}-(z-L)^{2}\right]^{1 / 2}
\end{aligned}
$$

Hence:

$$
\begin{aligned}
& P_{f b}(t)=\sum_{k=1}^{\infty} P_{f b k}(t) \\
& P_{f b}(t)=\exp \left\{-D_{f}\left[(4 \pi / 3) R^{3}+2 \pi L R^{2}\right]\right\}\left\{\exp \left[D_{f} C\left(R, D_{s}, L\right)\right]-1\right\}
\end{aligned}
$$

and finally:

$$
P_{f}(t)=\exp \left[-E_{f}(t)\right]
$$

with

$$
E_{f}(t)=D_{f}\left[(4 \pi / 3) R^{3}+2 \pi L R^{2}\right]-D_{f} C\left(R, D_{s}, L\right)
$$

$C\left(R, D_{s}, L\right)$ expressed by Eq. (9b) can be calculated either numerically or analytically. Depending on the position of the nanofiber center $(r, z)$ and $R$, entire fiber length $2 L$ or a nanofiber section, either $2\left(R^{2}-r^{2}\right)^{1 / 2}$ or $L-z+\left(R^{2}-r^{2}\right)^{1 / 2}$ is embedded in 
the sphere. This depends on the positions of nanofiber ends: $(r, z+L)$ and $(r, z-L)$ relative to the sphere surface. $R$ increases with time, hence three cases should be considered: $R \leqslant L, L \leqslant R \leqslant 2 L$ and $R \geqslant 2 L$, as shown in Fig. 1a-c. For the sake of brevity further derivations are presented in Appendix A. $C\left(R, D_{s}, L\right)$ is in the form:

For $R \leqslant L$ :

$$
C\left(R, D_{s}, L\right)=\left(2 \pi / D_{s}\right)\left\{R^{2}+\left[1-\exp \left(-2 D_{s} R\right)\right]\left[L /\left(2 D_{s}\right)-1 / D_{s}^{2}\right]+\exp \left(-2 D_{s} R\right)\left(R^{2}-R L+2 R / D_{s}\right)\right\}
$$

For $L \leqslant R \leqslant 2 L$ and $R \geqslant 2 L$

$$
\begin{aligned}
C\left(R, D_{s}, L\right)= & (2 \pi / 3)\left(2 R^{3}-3 R^{2} L+L^{3}\right) \exp \left(-2 D_{s} L\right)+\left(\pi / D_{s}^{2}\right)\left\{\left[1-\exp \left(-2 D_{s} L\right)\right]\left(2 D_{s} R^{2}-2 / D_{s}+L\right)+2 L\left(L D_{s}+2\right)\right. \\
& \left.\times \exp \left(-2 D_{s} L\right)\right\}
\end{aligned}
$$

Eqs. (12) and (13) were derived for all the nanofibers aligned in the same direction. Let us assume, however, that there are $m$ populations of nanofibers differently oriented, and the respective contents are denoted as $D_{f i}, i=1, \ldots, m$, where $\sum_{i=1}^{m} D_{f i}=D_{f}$. For each nanofiber population a separate zone around $A$ should be considered, with z-axis parallel to the orientation direction, and the probability $P_{f i}$ that no nucleation event will occur on the nanofibers of the respective population in the sphere around $A$, is expressed as:

$$
P_{f i}=\exp \left\{-D_{f i}\left[(4 \pi / 3) R^{3}+2 \pi L R^{2}\right]+D_{f i} C\left(R, D_{s}, L\right)\right\}
$$

The probability $P_{f}$ that no nucleation event will occur on any nanofiber in the sphere around $A$ is a product of all $P_{f i}$ and it is again expressed by Eq. (12), which is then valid regardless of nanofiber orientation.

Similar reasoning applies for $m$ populations of nanofibers with different nucleation abilities, reflected in different $D_{s i}, i=1, \ldots, m$, and contents $D_{f i}, i=1, \ldots, m$, where again $\sum_{i=1}^{m} D_{f i}=D_{f}$. In this case multiplication of the respective probabilities leads to the expression:

$$
P_{f}=\exp \left\{-D_{f}\left[(4 \pi / 3) R^{3}+2 \pi L R^{2}\right]+\sum_{i=1}^{m} D_{f i} C\left(R, D_{s i}, L\right)\right\}
$$

Finally, one can consider a nanofiber half-length distribution (by number), $D_{f}(L) d L$, for which multiplication of the respective probabilities yields:

$$
P_{f}(t)=\exp \left\{-\int_{0}^{\infty} D_{f}(L)\left[(4 \pi / 3) R^{3}+2 \pi L R^{2}\right] d L+\int_{0}^{\infty} D_{f}(L) C\left(R, D_{s}, L\right) d L\right\}
$$

It appears that according to Eq. (4) $\alpha$ is expressed as:

$$
\alpha(t)=1-P_{b}(t) P_{f}(t)
$$

and

$$
\alpha_{n}(t)=1-P_{b}(t)
$$

where $\alpha_{n}$ is the conversion degree in neat polymer, in the absence of nanofibers. Thus:

$$
\alpha(t)=1-\left[1-\alpha_{n}(t)\right] P_{f}(t)
$$

It also appears that $C\left(R, D_{s}, L\right) \rightarrow(4 \pi / 3) R^{3}+2 \pi L R^{2}$ for $D_{s} \rightarrow 0$. For a very dense nucleation, $D_{s} \rightarrow \infty$, leading to transcrystallinity, $C\left(R, D_{S}, L\right) \rightarrow 0$, hence:

$$
E_{f}(t)=D_{f}\left[(4 \pi / 3) R^{3}+2 \pi L R^{2}\right]
$$

For instantaneous nucleation in polymer bulk with density $D, P_{0}(t)$ is given by:

$$
P_{0}(t)=\exp \left[-(4 \pi / 3)\left(D R^{3}+D_{f} R^{3}\right)-2 D_{f} \pi L R^{2}+D_{f} C\left(R, D_{s}, L\right)\right]
$$

If instantaneous bulk nucleation is delayed by $\Delta t$ in respect to the nucleation on nanofibers, the term $D R^{3}$ should be replaced by $D \rho(\Delta t, t)^{3}$ with $\rho$ given by Eq. (3).

The equations derived above are valid for both isothermal and nonisothermal processes.

It can be noticed that for isothermal crystallization $(G=$ const) Eq. (20) yields:

$$
\ln \{-\ln [1-\alpha(t)]\}=\ln \left[(4 \pi / 3)(G t)^{3}\left(D+D_{f}\right)+2 \pi D_{f} L(G t)^{2}-D_{f} C\left(G t, D_{s}, L\right)\right]
$$

It is then clear that the Avrami analysis can yield a straight line when in the logarithm argument the second component dominates over the other two, that is when $D_{s}$ is very large decreasing $C\left(G t, D_{s}, L\right)$, nanofibers are long and the effect of nucleation at their ends is relatively small, and also bulk nucleation is weak. In that case the Avrami exponent value will be close to two. In the case of small $2 D_{f} L$ or $D_{s}$ the bulk nucleation will dominate, leading to the exponent value close to three. The case of spontaneous bulk nucleation with constant rate is even more complex, since $E_{b}(t)$ will be in the form of $K t^{4}$. Still, for large values of $2 D_{f} L$ and $D_{s}$ the Avrami exponent close to two can be predicted. 


\subsection{Extended volume approach}

In the derivation of $P_{f}$ it is necessary to calculate $C\left(R, D_{s}, L\right)$. It can be envisaged that the derivation will be much more complex, for instance, for curved nanofibers. However, it appears that the exponent in Eq. (20) is equal to the extended volume of spherulites on nucleating nanofibers, providing that the sum of extended volume of spherulites is replaced by an envelope covering all spherulites nucleated on the nanofiber (neglecting truncation due to impingement of spherulites nucleated in bulk and on other nanofibers), as shown in Fig. 2.

The envelope will be referred to as 'nanofiber extended volume' for short. Points at the distance $R$ from a nanofiber, which is a maximum spherulite radius of a spherulite at time $t$, are located on a surface of a cylinder of radius $R$ and length $2 L$, with the nanofiber in axis, and on surfaces of two hemispheres of radius $R$, with the fiber ends in centers, as shown in Fig. 3. Thus the maximum extended volume for a strongly nucleating nanofiber is equal to a volume of the cylinder and the two hemispheres, which leads to Eq. (19). For $D_{s} \rightarrow \infty$ the extended volume for a single nanofiber reaches its maximum value, whereas it is smaller for finite $D_{s}$. Since nucleation on nanofibers occurs randomly, nanofibers extended volumes differ and should be added. However, we can replace that sum by a product of $D_{f}$ and an average 'extended volume' calculated using probability theory. To this aim a part of the maximum extended volume unoccupied at time $t$ by spherulites has to be calculated and subtracted.

For each point $B(r, z)$ located within the maximum extended volume, where $r$ and $z$ are cylindrical coordinates (Fig. 3 ) the probability, $q_{1}(r, z)$ or $q_{2}(r, z)$, that it remains at time $t$ outside spherulites nucleated on the nanofiber is calculated, and integration is performed over the volume; only half of the cylinder and one hemisphere can be taken into account because of the symmetry. To simplify integration, different systems of coordinates are used for the cylinder and the hemisphere. The integrals, multiplied by 2 , are subtracted from the maximum extended volume:

$$
E_{f}(t)=D_{f}\left[(4 \pi / 3) R^{3}+2 \pi L R^{2}-4 \pi \int_{0}^{L} \int_{0}^{R} r q_{1}(r, z) d r d z-4 \pi \int_{0}^{R} \int_{0}^{R_{z}} r q_{2}(r, z) d r d z\right]
$$

with

$$
R_{z}=\left(R^{2}-z^{2}\right)^{1 / 2}
$$

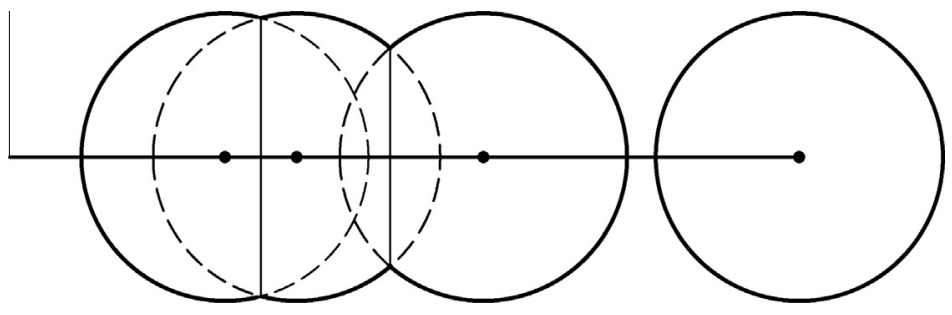

Fig. 2. 'Nanofiber extended volume' marked by thick solid line.

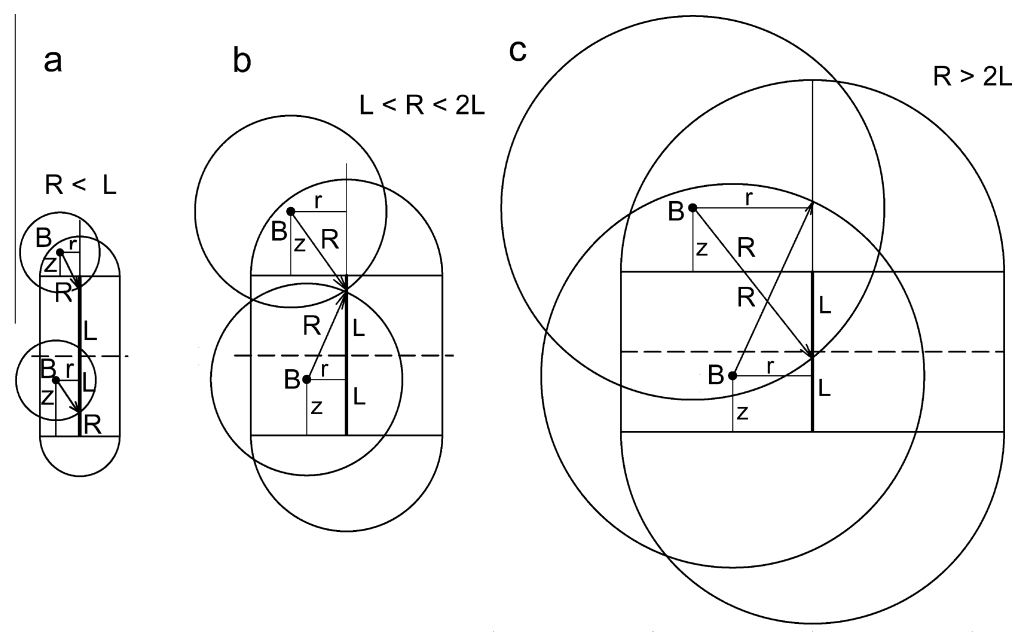

Fig. 3. Maximum extended volume for a straight nanofiber for different ratios between $R$ and $L$, and examples of positions of point $B$ within it. 
$q_{1}(r, z)$ and $q_{2}(r, z)$ are equal to $\exp \left[-D_{s} L_{s}(r, z)\right]$, where $L_{s}$ is the section of considered nanofiber embedded in a sphere around the point $B$ with the radius $R$, as shown in Fig. 3a-c. Depending on a position of $B$ and on $R, L_{s}$ is equal to $2 L, 2\left(R^{2}-r^{2}\right)^{1 / 2}$, $\left(R^{2}-r^{2}\right)^{1 / 2}+z$ or $\left(R^{2}-r^{2}\right)^{1 / 2}-z$. The detailed forms of the integrals on the right side of Eq. (22a), denoted as $S_{1}$ and $S_{2}$, respectively, are shown in Appendix B. As it was expected:

$$
S_{1}\left(R, D_{s}, L\right)+S_{2}\left(R, D_{s}, L\right)=C\left(R, D_{s}, L\right)
$$

Taking into account spherulites nucleated in the polymer bulk leads to $\alpha$ as expressed by an equation equivalent to Eq. (17a):

$$
\alpha(t)=1-\exp \left[-E_{b}(t)-E_{f}(t)\right]
$$

It is worth noting that no assumption about the nanofibers orientation is required to derive Eq. (24).

The exponent in Eq. (24) is a sum of extended volumes. A length distribution of nanofibers and/or different ability of nanofibers to nucleate matrix crystallization can be accounted for by summation or integration of respective extended volumes, which will lead to the same expressions as those derived in the previous section.

It is of interest that using this approach one can easily calculate the extended volume for nucleation on infinite length straight nanofibers:

$$
\begin{aligned}
& E_{f}(t)=D_{f l}\left\{\pi R^{2}-2 \pi \int_{0}^{R} r \exp \left[-2 D_{s}\left(R^{2}-r^{2}\right)^{1 / 2}\right] d r\right\} \\
& E_{f}(t)=D_{f l}\left\{\pi R^{2}-\left(\pi / 2 D_{s}^{2}\right)\left[1-\left(1+2 D_{s} R\right) \exp \left(-2 D_{s} R\right)\right]\right\}
\end{aligned}
$$

which permits to obtain the expression for $\alpha$ identical with that derived in [15], where $D_{f l}$ is the length of nanofibers per volume unit of a nanocomposite.

\section{Curved nanofibers}

As a model, a nanofiber in the form of half-circle with radius $R_{S}$ is considered (Fig. 4 ). $R_{S}=2 L / \pi$ for a nanofiber of length $2 L$. The extended volume for such nanofibers can be calculated using the concept of extended volume as for straight nanofibers. Points at a distance $R$ from the nanofiber are located on a surface of a half-toroid, along the nanofiber, with a radius $R$, and on two hemispheres with the nanofiber ends in the centers, also with radii $R$. Thus, the maximum extended volume for the nanofiber is equal to a volume of half-toroid plus two hemispheres, as shown in Fig. 4, excluding the overlapping parts. For each point $B$ in this volume, the probability that it remains at time $t$ unoccupied by spherulites nucleated on the nanofiber is calculated, $p_{1}(x, y, \gamma)$ and $p_{2}(x, y, z)$, and integration is performed over the half-toroid and hemispheres. To simplify integration, different systems of coordinates are used for the toroid and the hemisphere, as shown in Fig. 4a. The integrals are then subtracted from the maximum extended volume, that is a volume of the half-toroid plus two hemispheres.

For $R \leqslant R_{s}$ :

$$
E_{f c}(t)=D_{f}\left[\pi^{2} R_{s} R^{2}+(4 \pi / 3) R^{3}-4 \int_{0}^{\pi / 2} \int_{-R}^{R}\left(R_{s}+x\right) \int_{0}^{R_{x}} p_{1}(x, y, \gamma) d y d x d \gamma-4 \int_{0}^{R} \int_{-R_{z}}^{R_{z}} \int_{0}^{R_{g}} p_{2}(x, x, z) d y d x d z\right]
$$

For $R \geqslant R_{s}$ :

$$
\begin{aligned}
E_{f c}(t)= & D_{f}\left\{\left(\pi^{2} / 2\right) R_{s} R^{2}+(\pi / 3)\left[\left(R^{2}-R_{s}^{2}\right)^{1 / 2}\left(R_{s}^{2}+2 R^{2}\right)+3 R^{2} R_{s} \arcsin \left(R_{s} / R\right)+2 R^{3}+3 R_{s} R^{2}-R_{s}^{3}\right]\right. \\
& \left.-4 \int_{0}^{\pi / 2} \int_{-R_{s}}^{R}\left(R_{s}+x\right) \int_{0}^{R_{x}} p_{1}(x, y, \gamma) d y d x d \gamma-4 \int_{0}^{R_{f}} \int_{-R_{s}}^{R_{z}} \int_{0}^{R_{g}} p_{2}(x, y, z) d y d x d z-4 \int_{R_{f}}^{R_{z}} \int_{-R_{z}}^{R_{g}} p_{0}(x, y, z) d y d x d z\right\}
\end{aligned}
$$

where

$$
R_{x}=\left(R^{2}-x^{2}\right)^{1 / 2}, \quad R_{z}=\left(R^{2}-z^{2}\right)^{1 / 2}, \quad R_{g}=\left(R^{2}-z^{2}-x^{2}\right)^{1 / 2} \text { and } R_{f}=\left(R^{2}-R_{s}^{2}\right)^{1 / 2}
$$

The first integral in Eq. (26a) is over the upper part of the zone resulted from a revolution over $0 \leqslant \gamma \leqslant \pi / 2$ of the circle of a radius $R$, and with the center at a distance $R_{s}$ from the revolution axis, whereas the second integral is over the upper half of one hemisphere. Such integration ranges are sufficient because of symmetry and only multiplication by a factor of four is required. For $R>R_{s}$ in Eq. (26b), to eliminate overlapping, only a part of the revolving circle is considered ( $-R_{s} \leqslant x \leqslant R$ ) and also only that part of the hemisphere, which does not overlap with the second one, as shown in Fig. 4c. The probabilities $p_{1}(x, y, \gamma)$ and $p_{2}(x, y, z)$ are equal to $\exp \left(-D_{s} L_{c}\right)$, where $L_{c}$ denotes the length of arc embedded in a sphere of radius $R$ with a 


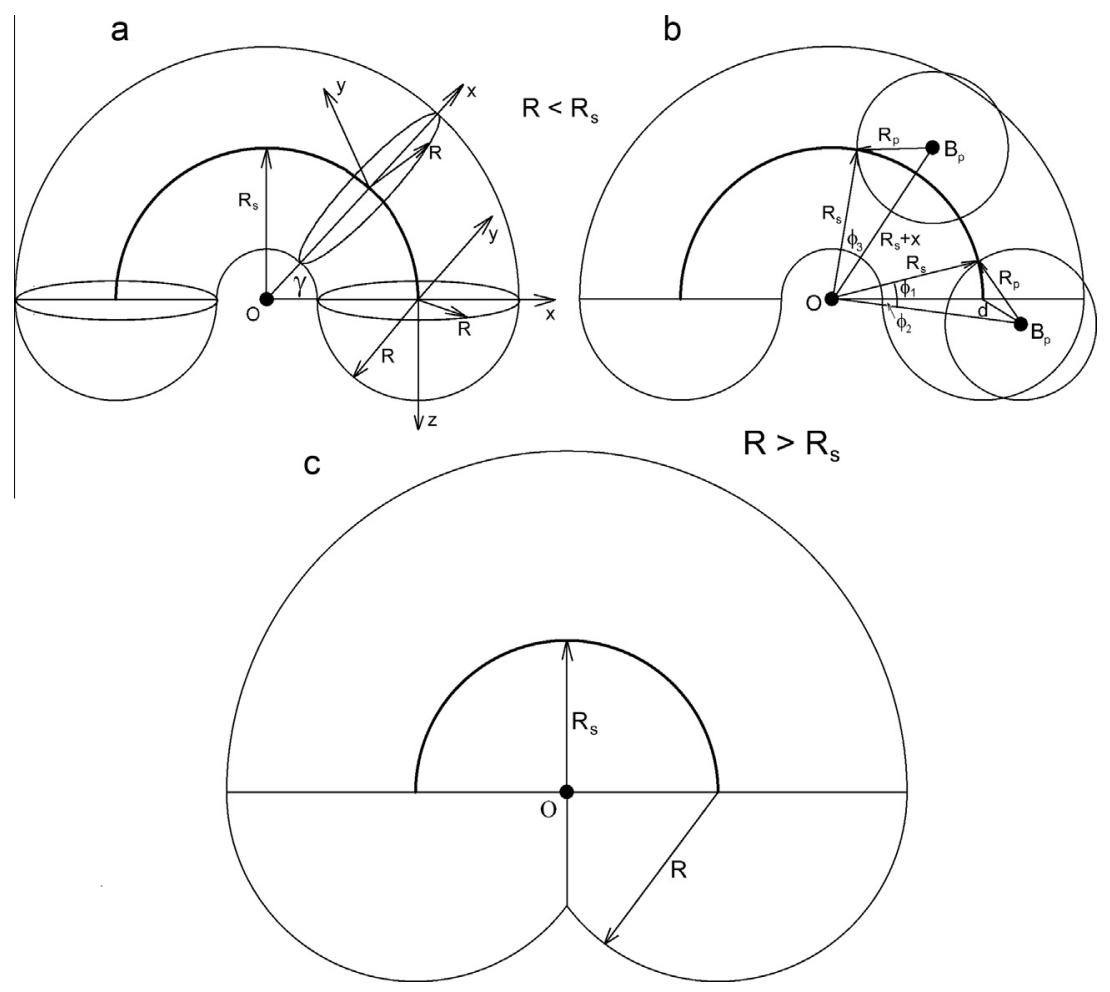

Fig. 4. Maximum extended volume for a half-circle nanofiber with systems of coordinates for integration over half-toroid and hemisphere (a), positions of point $B_{p}$ on plane $y=0$, and schemes for calculation the length of arcs included in the circle of radius $R_{p}$ (b), and scheme of the toroid for $R>R_{s}$ (c).

center at point $B$ at $(x, y, \gamma)$ or $(x, y, z)$, respectively, which can be equal to $2 L=\pi R_{s}$ or to its part, depending on the considered point position and on $R$, as illustrated in Fig. 4c. For the sake of brevity the more detailed expressions for $p_{1}(x, y, \gamma)$ and $p_{2}(x, y . z)$ are listed in Appendix C. $\alpha$ is expressed in the following way:

$$
\alpha(t)=1-\exp \left[-E_{b}(t)-E_{f c}(t)\right]
$$

Similarly to the case of straight nanofibers, no assumption concerning the nanofiber orientation was required to derive Eq. (27), and different ability to nucleate polymer crystallization by nanofibers of varying length can be accounted for as for straight nanofibers by expressing $E_{f c}(t)$ as an appropriate sum or integral. It is worth noting that the above derived equations, similarly to the case of straight nanofibers, contain $R$, which is equal to $G t$ for isothermal crystallization, whereas to the integral of $G$ over time for nonisothermal crystallization (Eq. (3)), and therefore these equations can be applied to both processes.

For $D_{s} \rightarrow \infty$ the integrals in Eqs. (26a) and (26b) vanish and $E_{f c}$ attains its maximum value. It appears that the first component in Eq. (26a) is equal to $2 \pi D_{f} L R^{2}$, hence for $D_{s} \rightarrow \infty$ Eq. (26a) is equivalent to Eq. (19). Moreover, numerical calculations showed that for $R>R_{s}$ maximum $E_{f c}$ calculated according to Eq. (26b) was smaller than $E_{f}$ expressed by Eq. (19) by less than five percent. It can be envisaged that the Avrami analysis of isothermal crystallization can yield a constant exponent close to two for $D_{s} \rightarrow \infty$ when the integrals vanish, and for large $D_{f}$ and $R_{s}$ (or $2 L$ ), similarly to the nucleation on straight nanofibers.

\section{Conversion degree components}

Based on Eqs. (17a) and (24), one can express the conversion rate, $d \alpha / d t$, in the form:

$$
\frac{d \alpha(t)}{d t}=\exp \left[-E_{b}(t)-E_{f}(t)\right] \frac{d E_{b}(t)}{d t}+\exp \left[-E_{b}(t)-E_{f}(t)\right] \frac{d E_{f}(t)}{d t}
$$

with $E_{b}$ and $E_{f}$ given by Eqs. (6a), (6b) and (12b). It can be demonstrated, similarly to the case of crystallization in a confined volume [35,36], where nucleation can occur inside a polymer and on the limiting surfaces, that the first and the second component of Eq. (28) describe the contributions from spherulites nucleated in polymer bulk and on nanofibers, respectively.

Integration over time leads to the components of $\alpha$ originating from spherulites nucleated in polymer bulk, $\alpha_{b}$, and on nanofibers, $\alpha_{f}$ : 


$$
\begin{aligned}
& \alpha_{b}(t)=\int_{0}^{t} \exp \left[-E_{b}(\tau)-E_{f}(\tau)\right] \frac{d E_{b}(\tau)}{d \tau} d \tau \\
& \alpha_{f}(t)=\int_{0}^{t} \exp \left[-E_{b}(\tau)-E_{f}(\tau)\right] \frac{d E_{f}(\tau)}{d \tau} d \tau
\end{aligned}
$$

Setting the upper limit of integration as $\infty$ allows to calculate final volume fractions of respective populations of spherulites. For instantaneous bulk nucleation $E_{b}$ is given by Eq. (6a) and

$$
\frac{d E_{b}(t)}{d t}=4 \pi D G(t) R^{2}
$$

whereas for nucleation on straight nanofibers, with $D_{s} \rightarrow \infty, E_{f}$ is given by Eq. (19), and

$$
\frac{d E_{f}(t)}{d t}=4 \pi D_{f} G(t)\left(R^{2}+L R\right)
$$

In the case of $D_{s} \rightarrow \infty$, one can calculate components originating from nucleation on nanofiber length and at its ends as integrals over time of $\exp \left[-E_{b}(t)-E_{f}(t)\right]$ multiplied by $4 \pi D G(t) R^{2}$ and $4 \pi D_{f} G(t) L R$, respectively.

\section{Computer simulation}

To verify the analytical expressions, especially Eqs. (12), (13), (22), (24), (26), and (27), computer simulation was performed using protocols developed previously [21,33]. The space coordinates of nanofibers centers, as well as those of nucleation sites of spherulites nucleated in bulk and on nanofibers were generated by a pseudorandom number generator. To avoid situation that each nanofiber will nucleate the same number of spherulites, all the centers were generated on a line of length equal to the total length of all nanofibers, and then they were assigned to respective nanofibers, in proper distances from nanofibers ends. The nanocomposites with nanofibers aligned in one direction and in three orthogonal directions were simulated for comparison, as well as nanofibers in the form of half-circles. The sizes of simulated samples, in the form of cubes, were adjusted to have at least 1000 spherulites. In order to determine the conversion degree, $10^{4}$ sampling pseudorandom points were generated, at distances from sample walls. The time of occlusion of each point by spherulites radially growing at a constant rate was determined. For visualization of the resulting structures, boundary points between neighboring spherulites were calculated in selected planes of the cubes. Arbitrary length unit (a.l.u.), arbitrary volume unit (a.v.u.) and arbitrary time unit (a.t.u.) were used. In all cases the constant spherulite growth rate was $G=1$ a.l.u/a.t.u.

\section{Model predictions and computer simulation results}

Figs. 5 and 6 show conversion degree curves and the Avrami plots, respectively, for nanocomposites with straight nanofibers 10 a.l.u. long, whereas crystallization half-time, $t_{h}$, and the Avrami exponent, $n$, values are listed in Table 1 .

Fig. 5 evidences a very good agreement between the results of computer simulation and predictions based on the analytical equations - the symbols fit to the lines. Practically the same results were obtained for nanofibers aligned in the same direction and for nanofibers aligned in three orthogonal directions, proving that orientation of nanofibers had no effect on overall crystallization kinetics, as predicted theoretically.

Fig. 5 shows that regardless of the presence or absence of bulk nucleation, crystallization accelerated with increasing nucleation density on fibers, $D_{s}$, and the nanofiber content, $D_{f}$. However, when high values of $D_{s}$ were reached, its further increase had no visible effect; the curves for $D_{s}=5$ a.l.u. ${ }^{-1}$ superimposed on the curves obtained for $D_{s} \rightarrow \infty$, that is for $E_{f}$ attaining its maximum value. Moreover, crystallization was faster for $D_{s}=0.1$ a.l.u. $^{-1}$ and $D_{f}=0.005$ a.v.u. $^{-1}$ than for $D_{s}=0.5$ a.l.u. $^{-1}$ and $D_{f}=0.001$ a.v.u. $^{-1}$, despite the same number of nucleation sites, showing that the increase of $D_{f}$ had a stronger effect on crystallization kinetics than the increase of $D_{s}$. This is because the spherulites nucleated on the same nanofiber are in close vicinity, which enhances truncation upon impingement. For the same reason crystallization for bulk nucleation with $D=0.001$ a.v.u. ${ }^{-1}$ in neat polymer is faster than in the case of nucleation occurring only on nanofibers, in the absence of bulk nucleation, despite the same number of nucleation sites, $2 L D_{f} D_{s}=0.001$ a.v.u. ${ }^{-1}$. In general, the presence of bulk nucleation accelerated crystallization compared to that in its absence but the effect diminished with increasing $D_{f}$ and $D_{s}$. It is worth noting that consideration of crystallization in the absence of bulk nucleation is justified because strongly nucleating nanofibers can exhibit activity at small undercoolings, where nucleation in neat polymer is negligibly weak.

Fig. 6 shows the Avrami plots for the data presented in Fig. 5. For small $D_{s}$ the Avrami plots resemble straight lines with the slopes, that is the exponent values, close to three. Increasing $D_{s}$ resulted in a slight departure from linearity and a decrease of the Avrami exponent, calculated for y-coordinate range of $2.97-1.10$ (corresponding to $0.05<\alpha<0.95$ ), to 2.2-2.4. In the case of 10 a.l.u. long nanofibers the smallest exponent value was obtained for $D_{s} \rightarrow \infty$ (that is for transcrystallinity) and the larger $D_{f}$. In the absence of bulk nucleation, for $D_{s} \rightarrow \infty$ and $D_{f}=0.001$ a.v.u. $^{-1}$ the slope of plot increased from 2.05 for $\alpha<0.05$ to 2.28 for $0.05<\alpha<0.95$. For $D_{f}=0.005$ a.v.u. ${ }^{-1}$ the respective values were 2.03 and 2.18 . It must be noted, however, that data for small $\alpha$ (and also small $1-\alpha$ ) are usually excluded from analysis because of too large experimental error [21,37]. Nevertheless, the increase of slope was caused by the component of $E_{f}$ proportional to $R^{3}$ (Eq. (19)) due 


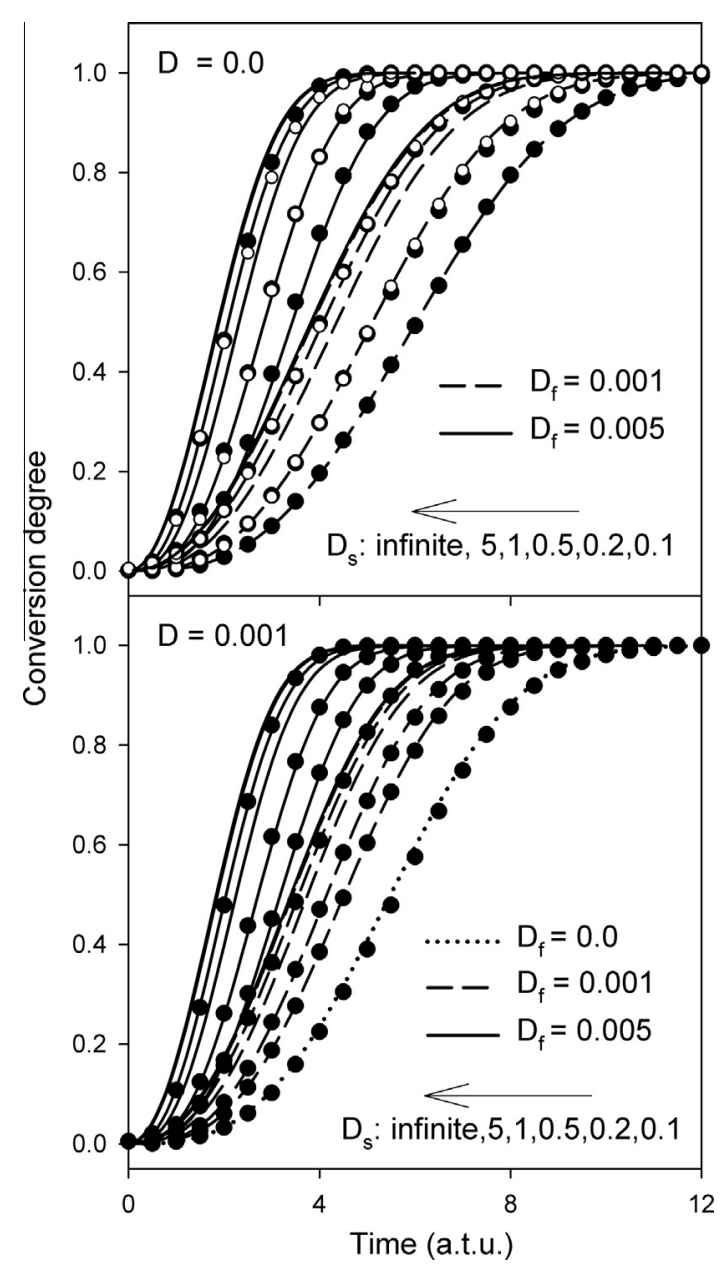

Fig. 5. Conversion degree vs. time for nanocomposites with different straight nanofiber contents, $D_{f}$, and nucleation densities, $D_{s}$, on nanofibers 10 a.l.u. long, in the absence or presence of instantaneous bulk nucleation with density $D$. Values of $D_{s}$ per a.l.u., $D_{f}$ and $D$ per a.v.u. indicated in the figure. Lines represent analytical results, whereas filled and empty symbols denote results of computer simulation for nanofibers aligned in the same direction and in three orthogonal directions, respectively.

to growth of spherulites nucleated at the nanofiber ends. In general, a change of $D_{f}$ should cause only a shift of the Avrami plot parallel to y-axis. However, due to the shift, different portions of the Avrami plots can fall in the considered data range, which can result in changes of the calculated exponent. This is illustrated on the example of the dash-dotted plot for $D_{f}=0.15$ a.v.u. ${ }^{-1}$ shown in Fig. 6 , for which the exponent for $0.05<\alpha<0.95$ was 2.04. Fig. 6 and Table 1 demonstrate that the presence of bulk nucleation can increase the Avrami exponent, but the effect diminishes with the increase of $D_{s}$ and $D_{f}$, as it was predicted by Eqs. (20) and (21).

Fig. 7 compares the conversion degree curves for crystallization nucleated on nanofibers with length of 10 and 20 a.l.u.; $t_{h}$ and the Avrami exponent values are listed in Table 1. To maintain the same $2 L D_{f}$ value, $D_{f}$ values for the latter were decreased twofold, from 0.001 to 0.0005 a.v.u. ${ }^{-1}$ and from 0.005 to 0.0025 a.v.u. ${ }^{-1}$. For the same $2 L D_{f}$ values the crystallization nucleated on the longer nanofibers was slower, and the difference increased with increasing $D_{s}$. The difference in $\alpha$ enlarged with time and was better visible after $t_{h}$. Thus, the increase of $D_{f}$ shortening the crystallization diminished the effect of nanofiber length, as illustrated in Fig. 7. The reason of slower crystallization was again an increased probability of nucleation of neighboring spherulites on the same nanofiber, in close vicinity, leading to enhanced truncation. For the same reason, the Avrami exponent decreased slightly, as it is detailed in Table 1, and the departure from linearity diminished, as shown in Fig. 8. In the case of transcrystallinity $\left(D_{s} \rightarrow \infty\right)$ and $D_{f}=0.0005$ a.v.u. ${ }^{-1}$ the exponent values were 2.03 and 2.20 for $\alpha<0.05$ and $0.05<\alpha<0.95$, respectively, whereas the respective numbers for $D_{f}=0.0025$ a.v.u. $^{-1}$ were 2.02 and 2.11. The decrease of the Avrami exponent compared to that calculated for transcrystallization nucleated on shorter fibers reflects the decreased effect of nanofiber ends, described by the term $(4 \pi / 3) D_{f} R^{3}$ in Eq. (21).

Fig. 9 shows time dependencies of conversion degree for crystallization nucleated on half-circle nanofibers, whereas $t_{h}$ and the Avrami exponent values are listed in Table 1. Symbols denoting results of computer simulation fit very well to 


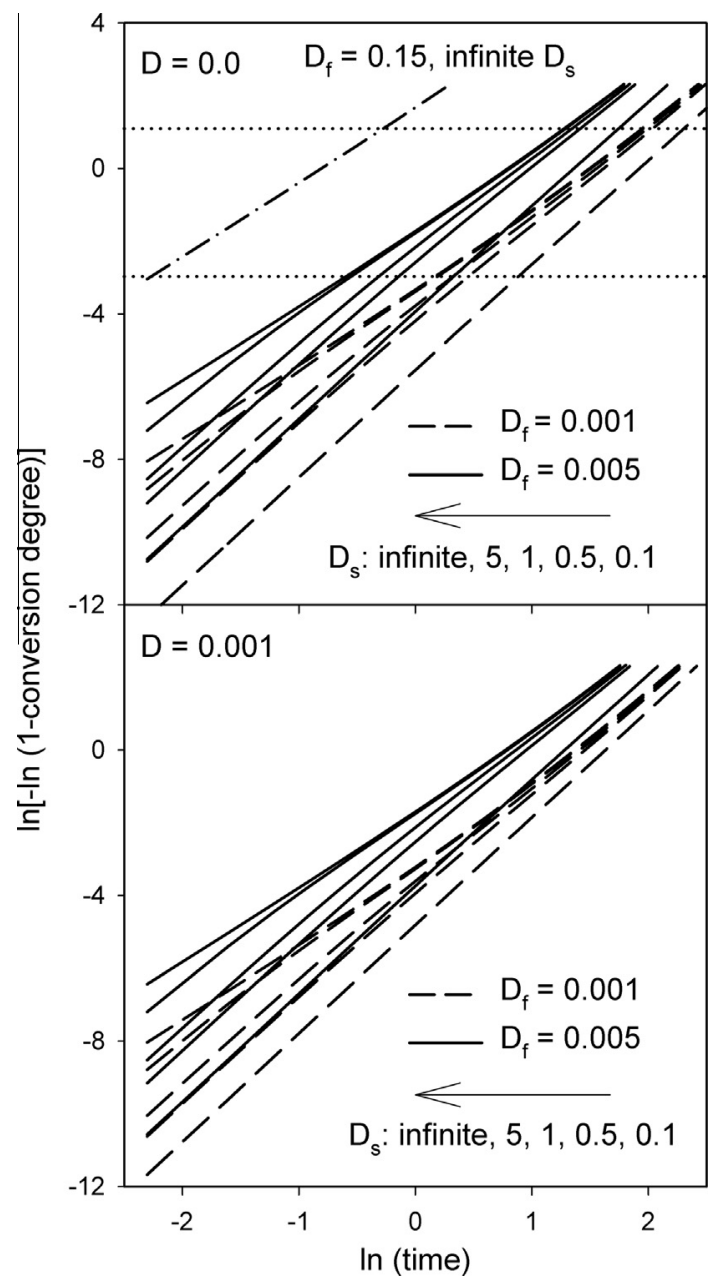

Fig. 6. The Avrami plots for nanocomposites with different straight nanofiber contents, $D_{f}$, and nucleation densities, $D_{s}$, on nanofibers 10 a.l.u. long, in the absence or presence of instantaneous bulk nucleation with density $D$. Values of $D_{s}$ per a.l.u., $D_{f}$ and $D$ per a.v.u. indicated in the figure. The dotted horizontal lines indicate the range of the Avrami y-coordinate for conversion degree ranging from 0.05 to 0.95 .

the lines drawn based on the expressions (26) and (27), confirming the correctness of the analytical model. It appears that the differences between the conversion degree for crystallization nucleated on the curved and straight nanofibers of the same length are insignificant, as it was already envisaged based on similarity of $E_{f}$ and $E_{f c}$ for $D_{s} \rightarrow \infty$. Moreover, the Avrami exponent values are practically the same as for crystallization nucleated on straight nanofibers provided that $L, D_{s}$ and $D_{f}$ parameters are identical.

Fig. 10 illustrates the spherulitic structures in nanocomposites with nucleating nanofibers in the absence (Fig. 10a, b, e, and f) and the presence (Fig. 10c and d) of bulk nucleation. The simulation was performed in 3D and the images are sections through the 3D cubes. All the interspherulitic boundaries are planar and seen as straight line sections because of instantaneous nucleation at the same time [38,39]. Dense nucleation on straight nanofibers (Fig. 10b, d, and e) led to formation of stacks of thin spherulites truncated by two boundaries parallel to each other and normal to nanofibers, which were formed due to impingement with neighbors nucleated on the same nanofibers. The nanocomposite shown in Fig. 10b is composed of such stacks and also of larger spherulites seen as polygons, which were nucleated on sites closest to nanofibers ends, with only one boundary normal to the nanofiber, formed with a neighboring spherulite nucleated on the same nanofiber. Those spherulites occupied 55\% of sample volume. It supports the observation that the increase of $D_{s}$ beyond a certain value has no effect on overall crystallization kinetics, due to strong truncation by the neighbors, and that the effect of nanofiber ends is of importance. In the case of weak nucleation on nanofibers (Fig. 10a) the stacks of sphelutites are absent, although neghboring spherulites nucleated on the same nanofibers with boundaries normal to the nanofibers are still visible.

The influence of bulk nucleation on the structure is illustrated in Fig. 10c and d. Fig. 10d clearly shows the presence of two populations of spherulites: nucleated on nanofibers and nucleated in bulk. The latter, of polygonal shape, with the 
Table 1

Crystallization half-time, $t_{h}$, and Avrami exponent, $n$, values for various nanofiber contents, $D_{f}$, nucleation densities on nanofibers, $D_{s}$, in the absence or presence of instantaneous bulk nucleation with density $D$. For calculation of the Avrami exponent conversion degree ranging from 0.05 to 0.95 was considered.

\begin{tabular}{|c|c|c|c|c|c|c|c|c|c|c|}
\hline $\mathrm{D}\left(\right.$ a.v.u $\left.{ }^{-1}\right)$ & $D_{f}\left(\right.$ a.v.u. $\left.{ }^{-1}\right)$ & 2L (a.l.u.) & $\mathrm{D}_{\mathrm{s}}\left(\right.$ a.l.u. $\left.{ }^{-1}\right)$ & $t_{h}$ (a.t.u.) & $\mathrm{n}$ & $D_{f}\left(\right.$ a.v.u. $\left.{ }^{-1}\right)$ & 2L (a.l.u.) & $\mathrm{D}_{\mathrm{s}}\left(\right.$ a.l.u. $\left.^{-1}\right)$ & $t_{h}$ (a.t.u.) & $\mathrm{n}$ \\
\hline 0.001 & 0.0 & 0 & 0.0 & 5.50 & 3.0 & - & - & - & - & - \\
\hline \multicolumn{11}{|c|}{ Straight nanofibers } \\
\hline \multirow[t]{5}{*}{0.001} & 0.001 & 10 & 0.1 & 4.53 & 2.9 & 0.005 & 10 & 0.1 & 3.18 & 2.9 \\
\hline & & & 0.5 & 4.16 & 2.9 & & & 0.5 & 2.26 & 2.6 \\
\hline & & & 1.0 & 3.75 & 2.7 & & & 1.0 & 2.04 & 2.5 \\
\hline & & & 5.0 & 3.57 & 2.6 & & & 5.0 & 1.88 & 2.2 \\
\hline & & & $\infty$ & 3.40 & 2.4 & & & $\infty$ & 1.85 & 2.2 \\
\hline \multirow[t]{5}{*}{0.0} & 0.001 & 10 & 0.1 & 6.03 & 2.9 & 0.005 & 10 & 0.1 & 3.43 & 2.9 \\
\hline & & & 0.5 & 4.37 & 2.6 & & & 0.5 & 2.32 & 2.6 \\
\hline & & & 1.0 & 4.08 & 2.4 & & & 1.0 & 2.09 & 2.4 \\
\hline & & & 5.0 & 3.86 & 2.3 & & & 5.0 & 1.91 & 2.2 \\
\hline & & & $\infty$ & 3.82 & 2.3 & & & $\infty$ & 1.88 & 2.2 \\
\hline \multirow[t]{5}{*}{0.0} & 0.0005 & 20 & 0.1 & 6.17 & 2.8 & 0.0025 & 20 & 0.1 & 3.46 & 2.8 \\
\hline & & & 0.5 & 4.59 & 2.4 & & & 0.5 & 2.36 & 2.5 \\
\hline & & & 1.0 & 4.35 & 2.3 & & & 1.0 & 2.14 & 2.3 \\
\hline & & & 5.0 & 4.19 & 2.2 & & & 5.0 & 2.00 & 2.1 \\
\hline & & & $\infty$ & 4.16 & 2.2 & & & $\infty$ & 1.98 & 2.1 \\
\hline \multicolumn{11}{|c|}{ Curved nanofibers } \\
\hline \multirow[t]{5}{*}{0.0} & 0.001 & 10 & 0.1 & 6.14 & 3.0 & 0.005 & 10 & 0.1 & 3.51 & 2.9 \\
\hline & & & 0.5 & 4.41 & 2.6 & & & 0.5 & 2.34 & 2.6 \\
\hline & & & 1.0 & 4.11 & 2.4 & & & 1.0 & 2.10 & 2.4 \\
\hline & & & 5.0 & 3.88 & 2.3 & & & 5.0 & 1.91 & 2.2 \\
\hline & & & $\infty$ & 3.83 & 2.3 & & & $\infty$ & 1.88 & 2.2 \\
\hline
\end{tabular}

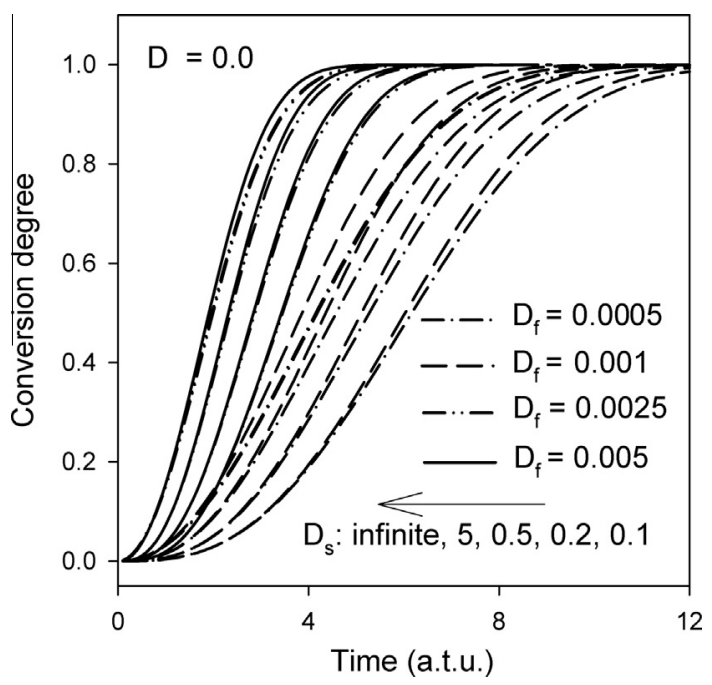

Fig. 7. Comparison of conversion degree vs. time for nanocomposites with different straight nanofiber contents, $D_{f}$, and nucleation densities, $D_{s}$, on nanofibers 10 and 20 a.l.u. long, in the absence of bulk nucleation $(D=0)$. Values of $D_{s}$ per a.l.u. and $D_{f}$ per a.v.u. indicated in the figure.

boundaries inclined at different angles to the nanofibers, separate the spherulitic stacks nucleated on the nanofibers. In the case of weak nucleation on nanofibers, the two populations of spherulites are less distinct, as shown in Fig. 10c; the presence of additional spherulites nucleated in bulk is mainly reflected in a decrease of spherulite sizes. Volume fractions of spherulites nucleated in bulk were 56 and 31\% (57 and 31\% according to Eq. (29a)) for samples shown in Fig. 10c and d, respectively. Interestingly, in the latter the volume fraction of spherulites nucleated closest to nanofiber ends was $36 \%$.

Fig. 10e illustrates the spherulitic structure nucleated on the nanofibers aligned in three orthogonal directions. Positions of the nanofibers normal to the figure plane are marked by dots. Although the overall crystallization kinetics does not depend on the orientation of nanofibers, the spherulitic patterns shown in Fig. 10b and e differ markedly. In the latter, stacks of spherulites nucleated on the nanofibers oriented in vertical and horizontal directions are seen, and also spherulites nucleated on the nanofibers perpendicular to the figure plane, visible as polygons, similarly to spherulites nucleated at the sites closest to nanofiber ends. 


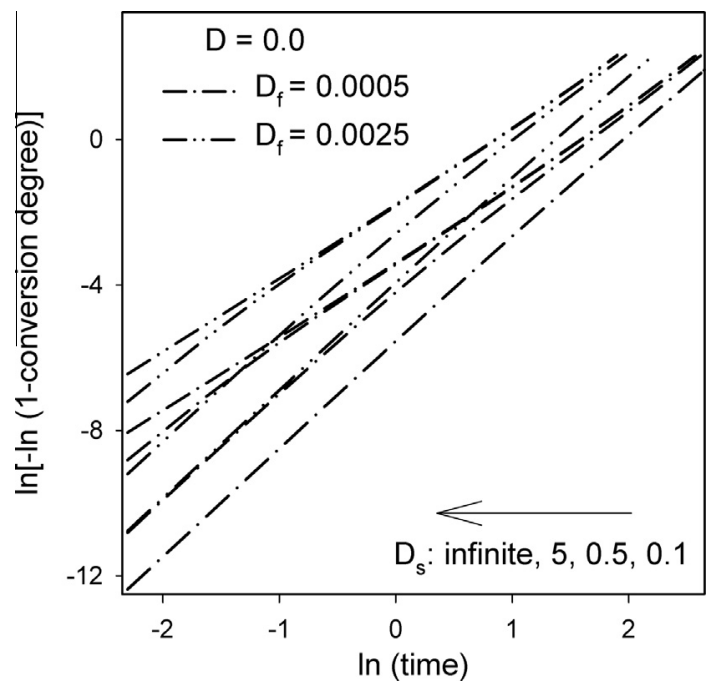

Fig. 8. The Avrami plots for nanocomposites with different straight nanofiber contents, $D_{f}$, and nucleation densities, $D_{s}$, on nanofibers 20 a.l.u. long, in the absence of bulk nucleation $(D=0)$. Values of $D_{s}$ per a.l.u. and $D_{f}$ per a.v.u. indicated in the figure.

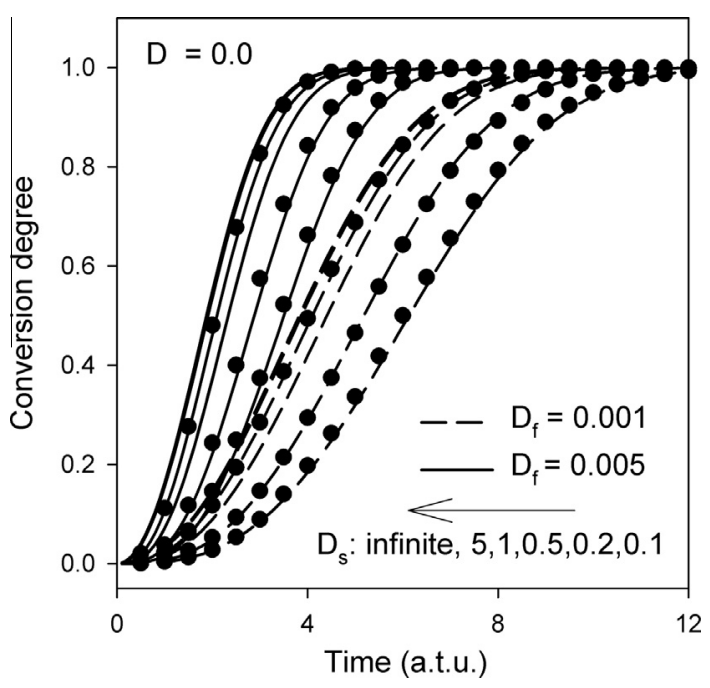

Fig. 9. Conversion degree vs. time for nanocomposites with different half-circle nanofiber contents, $D_{f}$, and nucleation densities, $D_{s}$, on nanofibers 10 a.l.u. long, in the absence of bulk nucleation $(D=0)$. Values of $D_{s}$ per a.l.u. and $D_{f}$ per a.v.u. indicated in the figure.

Fig. 10f illustrates the structure composed of spherulites nucleated on half-circle nanofibers. Both the analytical model and computer simulation demonstrated that the influence of the nanofiber shape on the overall crystallization kinetics was insignificant. However, the structure shown in Fig. 10f differs markedly from those presented in Fig. 10b and e. The stacks of spherulites nucleated on the same nanofibers are visible, limited by boundaries converging at common lines, passing through centers of half-circles, seen on the cross-section as points.

Although the model cases of nanocomposites with well oriented straight or half-circle nanofibers are idealized, similarly to the case with nanofibers oriented in three directions, those examples demonstrate that crystallization with similar, or even the same overall crystallization kinetics, can lead to very different spherulitic patterns.

\section{Crystallization kinetics of polypropylene reinforced with poly(tetrafluoroethylene) nanofibers}

It was shown recently that poly(tetrafluoroethylene), PTFE, nanofibers can be generated in situ by shearing of crystalline PTFE inclusions during compounding with another molten polymer and hence a nanocomposite can be obtained [40,41]. Here, polypropylene, PP, (Malen-P B200, Basell Orlen Polyolefins) with MFI of $0.6 \mathrm{~g} / 10$ min and zero shear viscosity of $4.19 \cdot 10^{4}$ Pa s at $170^{\circ} \mathrm{C}$, was melted in a Brabender internal mixer (W50E, Brabender GmbH, Duisburg, Germany). Then PTFE 

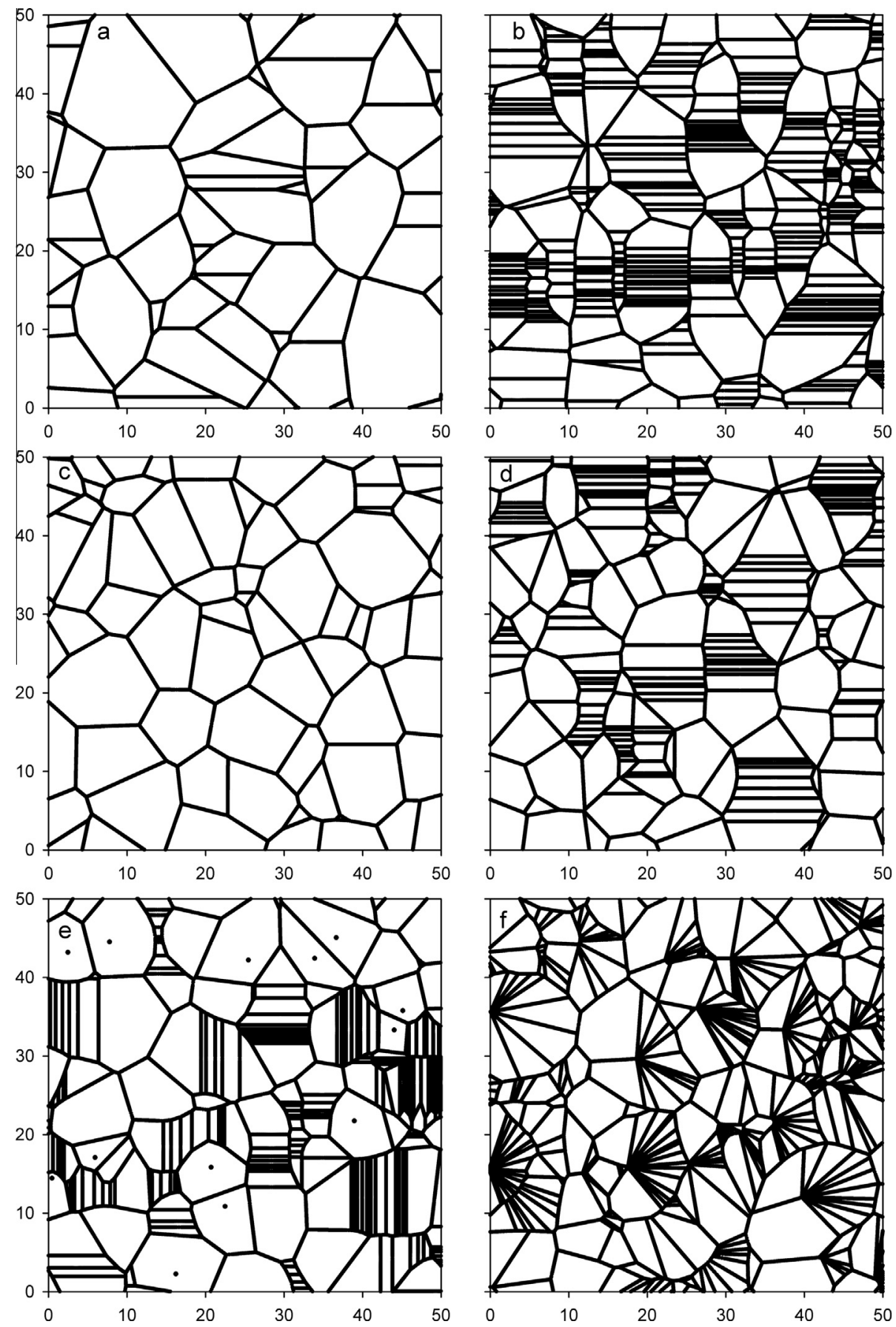

Fig. 10. Cross-sections, 50 a.l.u. $\times 50$ a.l.u., of simulated 3D nanocomposites with nanofiber content $D_{f}$ of 0.001 a.v.u. ${ }^{-1}$ : (a) - straight nanofibers aligned in vertical direction, $D=0, D_{s}=0.1$ a.l.u. $^{-1}$, (b) - straight nanofibers aligned in vertical direction, $D=0, D_{s}=1$ a.l.u. $^{-1}$, (c) - straight nanofibers aligned in vertical direction, $D=0.001$ a.v.u. ${ }^{-1}, D_{s}=0.1$ a.l.u. ${ }^{-1}$, (d) - straight nanofibers aligned in vertical direction, $D=0.001$ a.v.u. ${ }^{-1}, D_{s}=1$ a.l.u ${ }^{-1}$, (e) - straight nanofibers aligned in three orthogonal direction, horizontal, vertical and normal to the figure plane, $D=0, D_{s}=1$ a.l.u. $^{-1}$, (f) - half-circle nanofibers parallel to the figure plane with diameters aligned in vertical direction, $D=0, D_{s}=1$ a.l.u. $^{-1}$.

powder (Teflon PTFE 7C, $T_{m}$ of $346^{\circ} \mathrm{C}$, average grain size of $25 \mu \mathrm{m}$, by DuPont) was added to the molten PP in the amount of 5 wt.\%. The PTFE powder was compounded with PP at $200{ }^{\circ} \mathrm{C}$ and $120 \mathrm{rpm}$ for $15 \mathrm{~min}$. Maximum shear rate during compounding was 1150/s. Neat PP was processed at the similar conditions in order to obtain a material with the same thermo-mechanical history as the nanocomposite with PTFE. Scanning electron microscopy, SEM, of cryo-fractured bars of PP/PTFE nanocomposite indicated that PTFE powder grains were transformed to long nanofibers being 30-150 nm thick (see Fig. 11). 


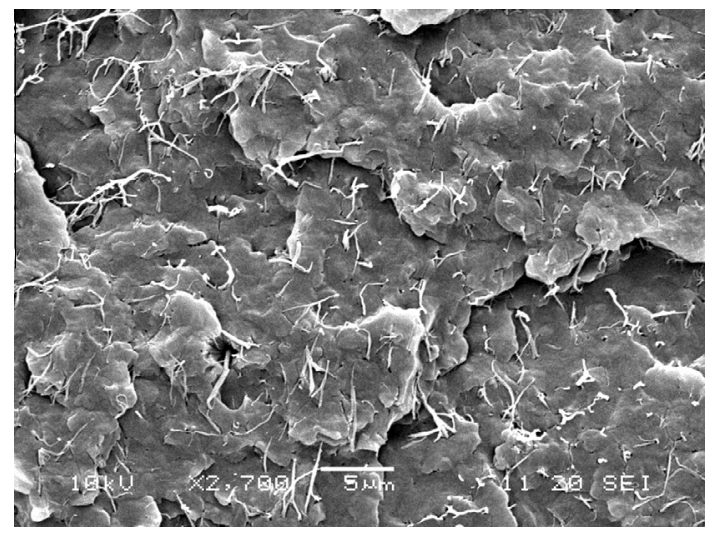

Fig. 11. SEM image of cryo-fractured surface of PP/PTFE nanocomposite prepared in Brabender internal mixer operating at $200{ }^{\circ} \mathrm{C}$ and $120 \mathrm{rpm}$ for 15 min.

Fig. 12 presents the Avrami plots for neat PP and PP/PTFE nanocomposite. Conversion degree, $\alpha(t)$, was calculated on the basis of differential scanning calorimetry, DSC (TA Q20, Thermal Analysis), thermograms recorded during isothermal crystallization. PP and PP/PTFE nanocomposite were annealed at $220^{\circ} \mathrm{C}$ for $3 \mathrm{~min}$ and cooled at $10{ }^{\circ} \mathrm{C} / \mathrm{min}$ to crystallization temperature of $128^{\circ} \mathrm{C}$ and $145^{\circ} \mathrm{C}$, respectively two different temperatures were chosen because the nanocomposite crystallized around $128{ }^{\circ} \mathrm{C}$ too fast to reach isothermal conditions, while at $145^{\circ} \mathrm{C}$ crystallization of neat PP was too long.

In the case of neat PP crystallized isothermally at $128^{\circ} \mathrm{C}$, the Avrami analysis of data for $\alpha$ in the range from 0.05 to 0.95 yields the exponent value of $2.90(\approx 3)$ indicating spherulitic crystallization originating from instantaneous nuclei. This is consistent with straight interspherulitic boundaries found in neat PP film.

Fig. 13 presents SEM (Jeol JSM-6000LA, Tokyo, Japan) microphotograph of free surface of $350 \mu \mathrm{m}$ thick film of PP/PTFE nanocomposite crystallized isothermally on a hot stage at $145^{\circ} \mathrm{C}$. The crystalline aggregates grew on PTFE nanofibers and assumed elongated shapes, reflecting underlying nanofibers. The presence of PTFE nanofibers induced the change of shape of growing polycrystalline aggregates from spherulitic to worm-like. In contrast to neat PP, the Avrami exponent value of $2.02(\approx 2)$ for PP/PTFE nanocomposite crystallized isothermally at $145^{\circ} \mathrm{C}$ indicated dense instantaneous nucleation on surfaces of PTFE nanofibers and rather low bulk nucleation density of PP as compared to the number of nuclei on PTFE nanofibers.

It can be concluded that PTFE nanofibers accelerated the crystallization of PP with simultaneous elevation of its crystallization temperature, changed the shape and the size of growing aggregates making them as long as PTFE nanofibers. The presence of PTFE nanofibers in PP matrix affected the kinetics of isothermal crystallization. The comparison with the prediction of the above outlined theory of crystallization kinetics led to the conclusion that the nucleation of PP crystals on PTFE nanofibers was very dense resembling transcrystallinity and Eq. (21), with $C\left(R, D_{s}, L\right) \rightarrow 0$ for very long and densely nucleating nanofibers, applies.

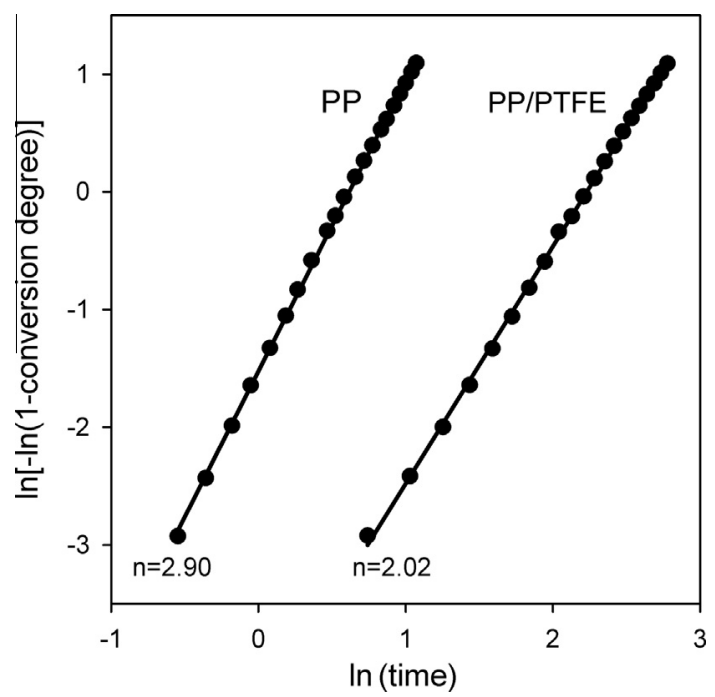

Fig. 12. The Avrami plots for isothermal crystallization of neat PP and PP/PTFE at $128{ }^{\circ} \mathrm{C}$ and $145^{\circ} \mathrm{C}$, respectively. Symbols - experimental data, lines - linear regression; $\alpha$ from the range of $0.05-0.95$. Time in min. 


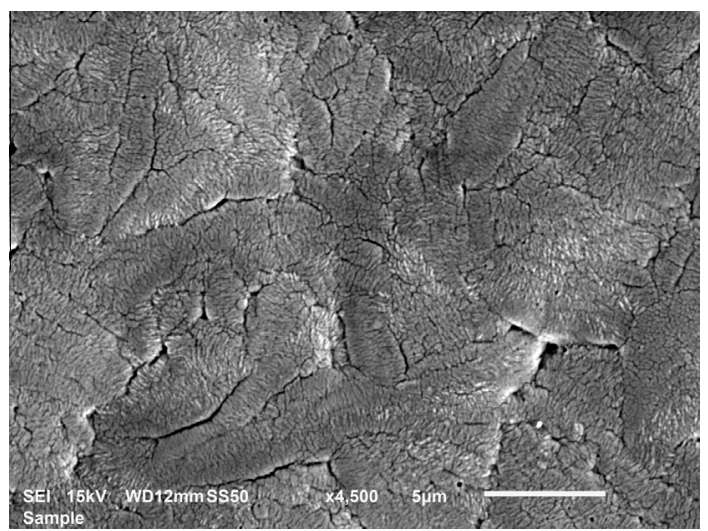

Fig. 13. SEM image of PP/PTFE nanocomposite film crystallized isothermally at $145{ }^{\circ} \mathrm{C}$ with free surface.
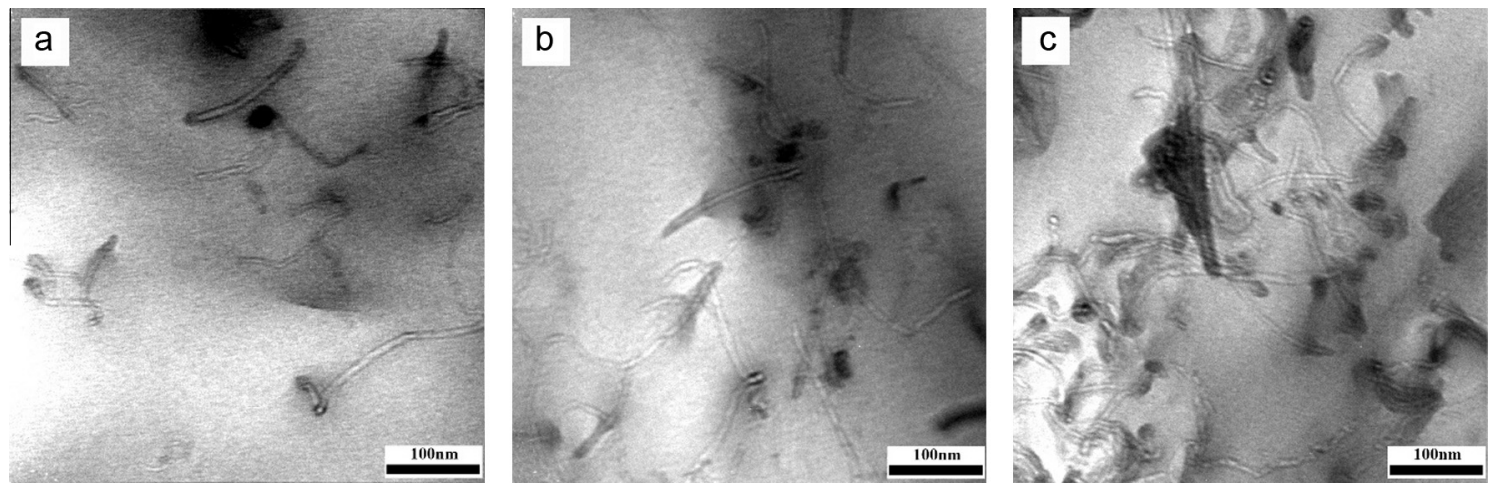

Fig. 14. TEM microphotographs of PA12 with (a) 0.67 wt.\%, (b) 2 wt.\%, (c) 4 wt.\% of MWCNTs.

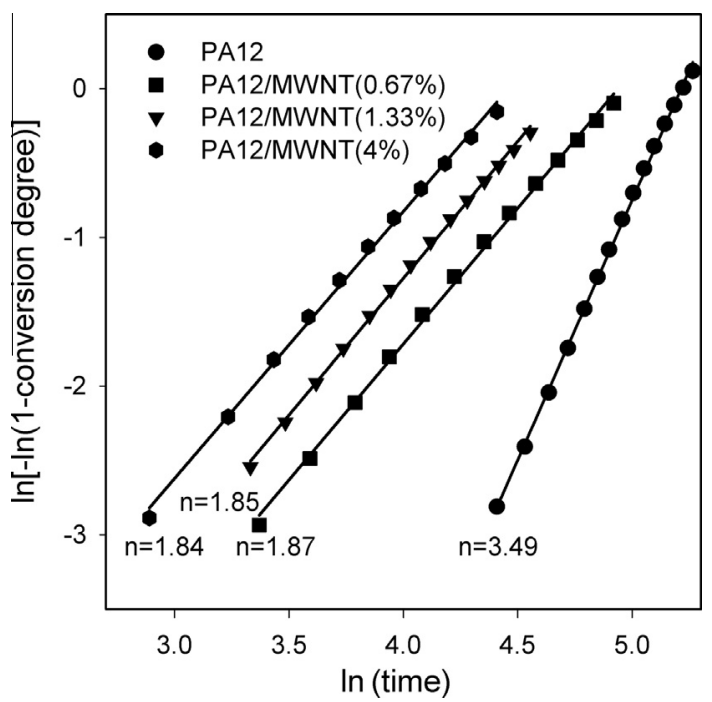

Fig. 15. The Avrami plots for neat PA12, and its nanocomposites with $0.67,1.33$ and 4 wt.\% of MWCNTs isothermally crystallized in a DSC at $172{ }^{\circ} \mathrm{C}$; symbols - experimental data, lines - linear regression; $\alpha(t)$ from the range of $0.05-0.95$. Time in min. 


\section{Crystallization kinetics of polyamide 12 reinforced with carbon nanotubes}

The matrix, polyamide 12, PA12, used was Rilsan ${ }^{\circledR}$ AMNO PA12 injection grade from Arkema, France. Its Newtonian viscosity is relatively low $\left(150 \mathrm{~Pa}\right.$ s at $\left.220^{\circ} \mathrm{C}\right)$. The temperatures of melting peak, $T_{m}$ of $178^{\circ} \mathrm{C}$, and crystallization peak, $T_{c}$ of $158{ }^{\circ} \mathrm{C}$, were measured by DSC. The multiwall carbon nanotubes, MWCNT, were provided by Arkema, in the form of a CM1-20 Graphistrength ${ }^{\circledR}$ masterbatch containing 20 wt.\% of MWCNTs dispersed in PA12. As produced, the MWCNTs had between 5 and 15 walls, outer diameters in the range of 10-15 nm and a maximum aspect ratio of 1000:1. Composite pellets with $0.67,1.33,2$ and 4 wt.\% of MWCNTs were first prepared by mixing the masterbatch with neat PA12 in a Brabender Plasti-Corder twin-screw extruder. The three heating zones were set at 190,210 and $220^{\circ} \mathrm{C}$ from the hopper to the nozzle. The screw rotation velocity was $50 \mathrm{rpm}$. The extrudate was stretched and cut in line with a Brabender Pelletizer CAN-plus. The MWCNTs contents were checked by thermogravimetric analysis (TGA) under air flow, from 20 to $600{ }^{\circ} \mathrm{C}$.

Isothermal crystallization of PA12 and PA12 nanocomposites was conducted in a DSC apparatus (TA Q20, Thermal Analysis). Samples of total mass of 6-8 mg were placed in aluminum pans, heated to $220^{\circ} \mathrm{C}$ and cooled to $172{ }^{\circ} \mathrm{C}$ at a constant rate of $10^{\circ} \mathrm{C} / \mathrm{min}$ under nitrogen flow. The dispersion of MWCNT was analyzed with a transmission electron microscope (TEM), Tesla BS 500 (Tesla, Czech Republic), operating at the accelerating voltage of $90 \mathrm{kV}$. Samples for TEM examination, in the form of ultra-thin sections $60 \mathrm{~nm}$ thick, were prepared by cryo-ultramicrotoming (PowerTome PC, Boeckeler, USA) equipped with a $35^{\circ}$ diamond knife (Diatome, Switzerland).

The dispersion of MWCNTs is illustrated in Fig. 14 showing the TEM micrographs for PA12/MWCNT systems with 0.67, 2 and 4 wt.\% of MWCNT.

The degree of dispersion of MWCNTs was good, especially in the case of system with low content of the nanofiller (0.67 wt.\%). In the case of higher content of MWCNT some nanotubes are interconnected, which promotes the formation of carbon nanotubes network.

The conversion degree, $\alpha(t)$, was calculated on the basis of DSC thermograms recorded during isothermal crystallization.

In the case of neat PA12, isothermally crystallized at $172^{\circ} \mathrm{C}$, the Avrami analysis of data for $\alpha(t)$ in the range from 0.05 to 0.95 yields the exponent value of 3.49 indicating spherulitic growth with slightly prolonged nucleation events (not fully instantaneous) (Fig. 15). However, for all the nanocomposites with MWCNTs the values of exponent were close to two, which indicates the growth of the polymer transcrystalline zones on carbon nanotubes nucleated instantaneously. Therefore, the presence of MWCNTs in PA12 matrix affects the kinetics of isothermal crystallization changing the slope of the Avrami plot. Similarly to PP/PTFE nanocomposites the prediction of the outlined theory of crystallization kinetics led to the conclusion that the nucleation of PA12 crystals on MWCNTs was very dense resembling transcrystallinity and the kinetics of crystallization as described by Eq. (21) with $C\left(R, D_{s}, L\right) \rightarrow 0$ for very dense nucleation on nanotube surfaces and very long nanotubes (aspect ratio 1000).

\section{Conclusions}

The model was developed describing the overall crystallization kinetics in nanofiber reinforced polymer nanocomposites. The two approaches were applied to nanocomposites with straight nucleating nanofibers: the first one taking advantage of the probability theory and the second one combining the extended volume approach and the probability theory. The second approach was used also to describe the overall crystallization kinetics in nanocomposites with curved nanofibers, in the form of half-circles. The results of analytical models were in good agreement with computer simulation. The derived mathematical formulas are in exponential forms suggesting the use of the Avrami logarithmic coordinates for detailed analysis of experimental data. The presence of nucleating nanofibers accelerated the crystallization depending, however, on their content, length, and nucleation ability. The increased probability of nucleation in close vicinity, on the same nanofiber, enhanced truncation and slowed down the crystallization. Due to that, crystallization nucleated on nanofibers was slower than nucleated in bulk on the same number of sites, also an increase of nanofiber length decelerated crystallization. Interestingly, the orientation of the nanofibers had no influence on overall crystallization kinetics, also the shape of nanofibers was found to have an insignificant effect despite the strong influence on the spherulitic patterns. The Avrami exponent decreased with increasing nanofiber content, their nucleation ability and length. The values close to two were calculated for nanocomposites with strongly nucleated nanofibers.

The elaborated approach can be used also for nanofibers with length distribution. Moreover, it is applicable not only to isothermal but also to nonisothermal conditions.

The prediction agreed well with the experimental results of examination of crystallization in polymer nanocomposites.

\section{Acknowledgement}

The project was financed from funds of the National Science Centre, Poland on the basis of the decision number DEC-2012/04/A/ST5/00606. 


\section{Appendix A}

For $R \leqslant L$ (Fig. 1a):

$$
\begin{aligned}
C\left(R, D_{s}, L\right)= & 4 \pi\left\{\int_{0}^{L-R} \int_{0}^{R} \exp \left[-2 D_{s}\left(R^{2}-r^{2}\right)^{1 / 2}\right] r d r d z+\int_{L-R}^{L} \int_{0}^{U} \exp \left\{-D_{s}\left[L-z+\left(R^{2}-r^{2}\right)^{1 / 2}\right]\right\} r d r d z\right. \\
& \left.+\int_{R}^{L} \int_{U}^{R} \exp \left[-2 D_{s}\left(R^{2}-r^{2}\right)^{1 / 2}\right] r d r d z+\int_{L}^{L+R} \int_{0}^{U} \exp \left\{-D_{s}\left[L-z+\left(R^{2}-r^{2}\right)^{1 / 2}\right]\right\} r d r d z\right\} \\
C\left(R, D_{s}, L\right)= & 4 \pi\left\{\int_{0}^{L-R} \int_{0}^{R} \exp \left[-2 D_{s}\left(R^{2}-r^{2}\right)^{1 / 2}\right] r d r d z+\int_{L-R}^{L+R} \int_{0}^{U} \exp \left\{-D_{s}\left[L-z+\left(R^{2}-r^{2}\right)^{1 / 2}\right]\right\} r d r d z\right. \\
& \left.+\int_{R}^{L} \int_{U}^{R} \exp \left[-2 D_{s}\left(R^{2}-r^{2}\right)^{1 / 2}\right] r d r d z\right\}
\end{aligned}
$$

where $U$ is given by Eq. (9c).

For $L \leqslant R \leqslant 2 L$ (Fig. $1 \mathrm{~b}$ ):

$$
\begin{aligned}
C\left(R, D_{s}, L\right)= & (2 \pi / 3)\left(2 R^{3}-3 R^{2} L+L^{3}\right) \exp \left(-2 D_{s} L\right)+4 \pi\left\{\int_{0}^{R-L} \int_{W}^{U} \exp \left\{-D_{s}\left[L-z+\left(R^{2}-r^{2}\right)^{1 / 2}\right]\right\} r d r d z\right. \\
& +\int_{0}^{R-L} \int_{U}^{R} \exp \left[-2 D_{s}\left(R^{2}-r^{2}\right)^{1 / 2}\right] r d r d z+\int_{R-L}^{L} \int_{0}^{U} \exp \left\{-D_{s}\left[L-z+\left(R^{2}-r^{2}\right)^{1 / 2}\right]\right\} r d r d z \\
& \left.+\int_{R-L}^{L} \int_{U}^{R} \exp \left[-2 D_{s}\left(R^{2}-r^{2}\right)^{1 / 2}\right] r d r d z+\int_{L}^{L+R} \int_{0}^{U} \exp \left\{-D_{s}\left[L-z+\left(R^{2}-r^{2}\right)^{1 / 2}\right]\right\}\right\} r d r d z \\
C\left(R, D_{s}, L\right)= & (2 \pi / 3)\left(2 R^{3}-3 R^{2} L+L^{3}\right) \exp \left(-2 D_{s} L\right)+4 \pi\left\{\int_{0}^{R-L} \int_{W}^{U} \exp \left\{-D_{s}\left[L-z+\left(R^{2}-r^{2}\right)^{1 / 2}\right]\right\} r d r d z\right. \\
& \left.+\int_{0}^{L} \int_{U}^{R} \exp \left[-2 D_{s}\left(R^{2}-r^{2}\right)^{1 / 2}\right] r d r d z+\int_{R-L}^{L+R} \int_{0}^{U} \exp \left\{-D_{s}\left[L-z+\left(R^{2}-r^{2}\right)^{1 / 2}\right]\right\}\right\} r d r d z
\end{aligned}
$$

where

$$
W=\left[R^{2}-(L+z)^{2}\right]^{1 / 2}
$$

For $R \geqslant 2 L$ (Fig. $1 \mathrm{c}$ ):

$$
\begin{aligned}
C\left(R, D_{s}, L\right)= & (2 \pi / 3)\left(2 R^{3}-3 R^{2} L+L^{3}\right) \exp \left(-2 D_{s} L\right)+4 \pi\left\{\int_{0}^{L} \int_{W}^{U} \exp \left\{-D_{s}\left[L-z+\left(R^{2}-r^{2}\right)^{1 / 2}\right]\right\} r d r d z\right. \\
& +\int_{0}^{L} \int_{U}^{R} \exp \left[-2 D_{s}\left(R^{2}-r^{2}\right)^{1 / 2}\right] r d r d z+\int_{L}^{R-L} \int_{W}^{U} \exp \left\{-D_{s}\left[L-z+\left(R^{2}-r^{2}\right)^{1 / 2}\right]\right\} r d r d z \\
& \left.+\int_{R-L}^{L+R} \int_{0}^{U} \exp \left\{-D_{s}\left[L-z+\left(R^{2}-r^{2}\right)^{1 / 2}\right]\right\}\right\} r d r d z \\
C\left(R, D_{s}, L\right)= & (2 \pi / 3)\left(2 R^{3}-3 R^{2} L+L^{3}\right) \exp \left(-2 D_{s} L\right)+4 \pi\left\{\int_{0}^{R-L} \int_{W}^{U} \exp \left\{-D_{s}\left[L-z+\left(R^{2}-r^{2}\right)^{1 / 2}\right]\right\} r d r d z\right. \\
& \left.+\int_{0}^{L} \int_{U}^{R} \exp \left[-2 D_{s}\left(R^{2}-r^{2}\right)^{1 / 2}\right] r d r d z+\int_{R-L}^{L+R} \int_{0}^{U} \exp \left\{-D_{s}\left[L-z+\left(R^{2}-r^{2}\right)^{1 / 2}\right]\right\}\right\} r d r d z
\end{aligned}
$$

The first components in Eqs. (A2a), (A2b), (A3a) and (A3b) originate from integration over the spherical cup: $0 \leqslant z \leqslant R-L$ and $0 \leqslant r \leqslant W$. If the fiber center is located within this cup, the entire fiber length $2 L$ is embedded in the sphere. Moreover the same expression for $C\left(R, D_{s}, L\right)$ is obtained for $L \leqslant R \leqslant 2 L$ and for $R \geqslant 2 L$. To verify correctness of integration numerical integration was performed. The values of $C\left(R, D_{s}, L\right)$ calculated based on Eqs. (A1)-(A3) and expressed by Eqs. (13a) and (13b) for various values of $R, L$ and $D_{s}$ agreed with those obtained by numerical integration.

\section{Appendix B}

For $R \leqslant L$ (Fig. 3a) $S_{1}$ and $S_{2}$ can be written in the form:

$$
S_{1}\left(R, D_{s}, L\right)=4 \pi \int_{0}^{R}\left\{\int_{0}^{R_{z}} \exp \left\{-D_{s}\left[\left(R^{2}-r^{2}\right)^{1 / 2}+z\right]\right\} r d r+\int_{R_{z}}^{R} \exp \left[-2 D_{s}\left(R^{2}-r^{2}\right)^{1 / 2}\right] r d r\right\} d z+4 \pi \int_{R}^{L} \int_{0}^{R} \exp \left[-2 D_{s}\left(R^{2}-r^{2}\right)^{1 / 2}\right] r d r d z
$$




$$
S_{2}\left(R, D_{s}, L\right)=4 \pi \int_{0}^{R} \int_{0}^{R_{z}} \exp \left\{-D_{s}\left[\left(R^{2}-r^{2}\right)^{1 / 2}-z\right]\right\} r d r d z
$$

where $R_{z}$ is given by Eq. (22b).

For $L \leqslant R \leqslant 2 L$ (Fig. 3b):

$$
\begin{aligned}
S_{1}\left(R, D_{s}, L\right)= & 4 \pi \int_{0}^{2 L-R}\left\{\int_{0}^{R_{z}} \exp \left\{-D_{s}\left[\left(R^{2}-r^{2}\right)^{1 / 2}+z\right]\right\} r d r+\int_{R_{z}}^{R} \exp \left[-2 D_{s}\left(R^{2}-r^{2}\right)^{1 / 2}\right] r d r\right\} d z \\
& +4 \pi \int_{2 L-R}^{L}\left\{\int_{0}^{H_{1}} \exp \left(-2 D_{s} L\right) r d r+\int_{H_{1}}^{R_{z}} \exp \left\{-D_{s}\left[\left(R^{2}-r^{2}\right)^{1 / 2}+z\right]\right\} r d r+\int_{R_{z}}^{R} \exp \left[-2 D_{s}\left(R^{2}-r^{2}\right)^{1 / 2}\right] r d r\right\} d z
\end{aligned}
$$

where

$$
H_{1}=\left[R^{2}-(2 L-z)^{2}\right]^{1 / 2}
$$

$S_{2}\left(R, D_{s}, L\right)$ is given by Eq. (A4b).

For $R \geqslant 2 L$ (Fig. 3c):

$$
\begin{aligned}
& S_{1}\left(R, D_{s}, L\right)=4 \pi \int_{0}^{L}\left\{\int_{0}^{H_{1}} \exp \left(-2 D_{s} L\right) r d r+\int_{H_{1}}^{R_{z}} \exp \left\{-D_{s}\left[\left(R^{2}-r^{2}\right)^{1 / 2}+z\right]\right\} r d r+\int_{R_{z}}^{R} \exp \left[-2 D_{s}\left(R^{2}-r^{2}\right)^{1 / 2}\right] r d r\right\} d z \\
& S_{2}\left(R, D_{s}, L\right)=4 \pi \int_{0}^{R}\left\{\int_{0}^{H_{2}} \exp \left(-2 D_{s} L\right) r d r+\int_{H_{2}}^{R_{z}} \exp \left\{-D_{s}\left[\left(R^{2}-r^{2}\right)^{1 / 2}-z\right]\right\} r d r\right\} d z
\end{aligned}
$$

where

$$
H_{2}=\left[R^{2}-(2 L+z)^{2}\right]^{1 / 2}
$$

\section{Appendix C}

For a nanofiber in the shape of half-circle, the easiest way to calculate the length of nanofiber arc embedded in a sphere of radius $R$ around a certain point $B$, seems to be determining an angle corresponding to the arc included in a circular section of the sphere in the nanofiber plane that is $y=0$, with the center at the point $B_{p}$, which is a projection of the point $B$ on this plane. For the point $B$ located at the coordinate $y$ the radius of this circle, $R_{p}$, is equal to $\left(R^{2}-y^{2}\right)^{1 / 2}$ (Fig. $\left.4 \mathrm{~b}\right)$. Knowing the distances from the center of the circle to the point $O$ where the axis of the toroid crosses the plane $y=0$, and to the fiber end, the latter required in the case of the points within the hemisphere, one can calculate the respective angles taking advantage of the law of cosines. For the points within the hemisphere (Fig. $4 \mathrm{~b}$ ):

$$
\begin{aligned}
& p_{2}(x, y, z)=\exp \left(-D_{s} R_{s} \varphi\right) \\
& \text { where for }\left(R^{2}-y^{2}\right)^{1 / 2} \leqslant R_{s}+d: \\
& \varphi=\varphi_{1}-\varphi_{2} \text { for } \varphi_{1}+\varphi_{2} \leqslant \pi \\
& \varphi=2 \varphi_{1}-\pi \text { for } \varphi_{1}+\varphi_{2} \geqslant \pi \\
& \varphi_{1}=\arccos \left[\left(x^{2}+y^{2}+z^{2}+2 R_{s}^{2}+2 x R_{s}-R^{2}\right) /\left(2 R_{s} d\right)\right] \\
& \varphi_{2}=\arccos \left[\left(R_{s}+x\right) / d\right] \\
& d=\left[\left(x+R_{s}\right)^{2}+z^{2}\right]^{1 / 2}
\end{aligned}
$$

If the condition (A7b) is not fulfilled $\varphi_{1}$ cannot be calculated because the circle with the radius $R_{\mathrm{p}}$ confines the circle with the radius $R_{S}$, hence the entire nanofiber. Therefore,

$$
\varphi=\pi \text { for }\left(R^{2}-y^{2}\right)^{1 / 2}>R_{s}+d
$$

For the points in the half-toroid, at $\gamma$ up to $\pi / 2$ (Fig. $4 \mathrm{~b}$ ):

$$
p_{1}(x, y, \gamma)=\exp \left(-D_{s} R_{s} \varphi\right)
$$


where for $\left(R^{2}-y^{2}\right)^{1 / 2} \leqslant 2 R_{s}+x$ :

$\varphi=2 \varphi_{3}$ for $\quad \varphi_{3} \leqslant \gamma$

$\varphi=\varphi_{3}+\gamma$ for $\pi-\gamma \geqslant \varphi_{3} \geqslant \gamma$

$\varphi=\pi$ for $\varphi_{3} \geqslant \pi-\gamma$

$\varphi_{3}=\arccos \left\{\left(x^{2}+y^{2}+2 R_{s}^{2}+2 x R_{s}-R^{2}\right) /\left[2 R_{s}\left(R_{s}+x\right)\right]\right\}$

If the condition (A8b) is not fulfilled, $\varphi_{3}$ cannot be determined since the circle of the radius $R_{p}$ confines the circle of the radius $R_{s}$. Hence:

$$
\varphi=\pi \text { for }\left(R^{2}-y^{2}\right)^{1 / 2}>2 R_{s}+x
$$

\section{References}

[1] L. Li, C.Y. Li, Ch. Ni, L. Rong, B. Hsiao, Structure and crystallization behavior of Nylon 66/multi-walled carbon nanotube nanocomposites at low carbon nanotube contents, Polymer 48 (2007) 3452-3460.

[2] J.Y. Kim, H.S. Park, S.H. Kim, Unique nucleation of multi-walled carbon nanotube and poly(ethylene 2,6-naphthalate) nanocomposites during nonisothermal crystallization, Polymer 47 (2006) 1379-1389.

[3] M. Razavi-Nouri, M. Ghorbanzadeh-Ahangari, A. Fereidoon, M. Jahanshahi, Effect of carbon nanotubes content on crystallization kinetics and morphology of polypropylene, Polym. Test. 28 (2009) 46-52.

[4] W. Hao, W. Yang, H. Cai, Y. Huang, Non-isothermal crystallization kinetics of polypropylene/silicon nitride nanocomposites, Polym. Test. 29 (2010) $527-533$.

[5] G.Z. Papageorgiou, D.S. Achilias, S. Nanaki, T. Beslikas, D. Bikiaris, PLA nanocomposites: Effect of filler type on non-isothermal crystallization, Thermochim. Acta 511 (2010) 129-139.

[6] C.A. Mitchell, R. Krishnamoorti, Non-isothermal crystallization of in situ polymerized poly(E-caprolactone) functionalized-SWNT nanocomposites, Polymer 46 (2005) 8796-8804.

[7] E.C. Chen, T.M. Wu, Isothermal crystallization kinetics and thermal behavior of poly(E-caprolactone)/multi-walled carbon nanotube composites, Polym. Deg. Stab. 92 (2007) 1009-1015.

[8] X. Yuan, C. Li, G. Guan, X. Liu, Y. Mao, D. Zhang, Synthesis and characterization of poly(ethylene terephthalate)/attapulgite nanocomposites, J. Appl. Polym. Sci. 103 (2007) 1279-1286.

[9] X. Chen, J. Xu, H. Lu, Y. Yang, Isothermal crystallization kinetics of poly(butylene terephthalate)/attapulgite nanocomposites, J. Polym. Sci., Part B: Polym. Phys. 44 (2006) 2112-2121.

[10] G. Sun, G. Chen, Z. Liu, M. Chen, Preparation, crystallization, electrical conductivity and thermal stability of syndiotactic polystyrene/carbon nanotube composites, Carbon 48 (2010) 1434-1440.

[11] R. Andrews, M.C. Weisenberger, Carbon nanotube polymer composites, Curr. Opin. Solid State Mater. Sci. 8 (2004) 31-37.

[12] J.N. Coleman, U. Khan, W.J. Blau, Y.K. Gun'ko, Small but strong: A review of the mechanical properties of carbon nanotube-polymer composites, Carbon 44 (2006) $1624-1652$.

[13] K. Chrissafis, K.M. Paraskevopoulos, A. Jannakoudakis, T. Beslikas, D. Bikiaris, Oxidized multiwalled carbon nanotubes as effective reinforcement and thermal stability agents of poly(lactic acid) ligaments, J. Appl. Polym. Sci. 118 (2010) 2712-2721.

[14] Z.M. Huang, Y.Z. Zhang, M. Kotaki, S. Ramakrishna, A review on polymer nanofibers by electrospinning and their applications in nanocomposites, Compos. Sci. Technol. 63 (2003) 2223-2253.

[15] K. Bernland, P. Smith, Nucleating polymer crystallization with poly(tetrafluoroethylene) nanofibrils, J. Appl. Polym. Sci. 114 (2009) $281-287$.

[16] E. Hammel, X. Tang, M. Trampert, T. Schmitt, K. Mauthner, A. Eder, P. Pötschke, Carbon nanofibers for composite applications, Carbon 42 (2004) $1153-$ 1158.

[17] K. Lozano, E.V. Barrera, Nanofiber-reinforced thermoplastic composites. I. Thermoanalytical and mechanical analyses, J. Appl. Polym. Sci. 79 (2001) $125-133$.

[18] M. Avrami, Kinetics of phase change. I. General theory, J. Chem. Phys. 7 (1939) 1103-1112.

[19] M. Avrami, Kinetics of phase change. II. Transformation-time relations for random distribution of nuclei, J. Chem. Phys. 8 (1940) $212-224$.

[20] M. Avrami, Kinetics of phase change. III. Granulation, phase change and microstructure, J. Chem. Phys. 9 (1941) 177-184.

[21] E. Piorkowska, A. Galeski, J.M. Haudin, Critical assessment of overall crystallization theories and predictions, Prog. Polym. Sci. 31 (2006) 549-575,

[22] E. Piorkowska, A. Galeski, Overall crystallization kinetics, in: E. Piorkowska, G.C. Rutledge (Eds.), Handbook of Polymer Crystallization, Wiley, Hoboken, NJ, 2013, pp. 215-235.

[23] U.R. Evans, The laws of expanding circles and spheres in relation to the lateral growth of surface films and the grain-size of metals, Trans. Faraday Soc. 41 (1945) 365-375.

[24] N. Billon, J.M. Escleine, J.M. Haudin, Isothermal crystallization kinetics in a limited volume. A geometrical approach based on Evans' theory, Colloid Polym. Sci. 267 (1989) 668-680.

[25] T. Ozawa, Kinetics of non-isothermal crystallization, Polymer 12 (1971) 150-158.

[26] E. Piorkowska, A. Galeski, Growth sites in space and time, J. Phys. Chem. 89 (1985) 4700-4703.

[27] E. Piorkowska, Modeling of polymer crystallization in a temperature gradient, J. Appl. Polym. Sci. 86 (2002) 1351-1362.

[28] L. Jarecki, B. Misztal-Faraj, Kinetic model of polymer crystallization with the lamellar thickness distribution, Eur. Polym. J. 73 (2015) 175-190.

[29] E. Piorkowska, Crystallization in polymer composites and nanocomposites, in: E. Piorkowska, G.C. Rutledge (Eds.), Handbook of Polymer Crystallization, Wiley, Hoboken, NJ, 2013, pp. 379-397.

[30] N.A. Mehl, L. Rebenfeld, Computer simulation of crystallization kinetics and morphology in fiber-reinforced thermoplastic composites. I. Twodimensional case, J. Polym. Sci., Part B: Polym. Phys. 31 (1993) 1677-1686.

[31] N.A. Mehl, L. Rebenfeld, Computer simulation of crystallization kinetics and morphology in fiber-reinforced thermoplastic composites. II. Threedimensional case, J. Polym. Sci., Part B: Polym. Phys. 31 (1993) 1687-1693.

[32] T.H. Krause, G. Kalinka, C. Auer, G. Hinrichsen, Computer simulation of crystallization kinetics in fiber-reinforced composites, J. Appl. Polym. Sci. 51 (1994) 399-406.

[33] E. Piorkowska, Modeling of crystallization kinetics in fiber reinforced composites, Macromol. Symp. 169 (2001) 143-148.

[34] A. Benard, S.G. Advani, An analytical model for spherulitic growth in fiber-reinforced polymers, J. Appl. Polym. Sci. 70 (1998) 1677-1687. 
[35] E. Piorkowska, Nonisothermal crystallization of polymers in samples of finite dimensions - 1. Background of the mathematical description of spherulitic pattern formation, Colloid Polym. Sci. 275 (1997) 1035-1045.

[36] E. Piorkowska, Nonisothermal crystallization of polymers in samples of finite dimensions - 2. Description of spherulitic pattern formation, Colloid Polym. Sci. 275 (1997) 1046-1059.

[37] A.T. Lorenzo, M.L. Arnal, J. Albuerne, A.J. Muller, DSC isothermal polymer crystallization kinetics measurements and the use of the Avrami equation to fit the data: Guidelines to avoid common problems, Polym. Test. 26 (2007) 222-231.

[38] T. Pakula, A. Galeski, E. Piorkowska, M. Kryszewski, Method of determining the kinetics of spherulite primary nucleation from the truncation of spherulites, Polym. Bull. 1 (1979) 275-279.

[39] A. Galeski, E. Piorkowska, Method of determining the kinetics of spherulite primary nucleation from the spherulite shapes in bulk samples, Polym. Bull. 2 (1980) $1-6$.

[40] K. Jurczuk, A. Galeski, E. Piorkowska, All-polymer nanocomposites with nanofibrillar inclusions generated in situ during compounding, Polymer 54 (2013) 4617-4628.

[41] K. Jurczuk, A. Galeski, E. Piorkowska, Strain hardening of molten thermoplastic polymers reinforced with poly(tetrafluoroethylene) nanofibers, J. Rheol. 58 (2014) 589-605. 